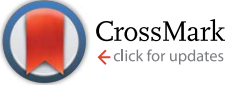

Cite this: RSC Adv., 2017, 7, 8323

Received 16th December 2016 Accepted 18th January 2017

DOI: $10.1039 / c 6 r a 28299 g$

www.rsc.org/advances

\section{Pyridine C3-arylation of nicotinic acids accessible via a multicomponent reaction: an entry to all- substituted-3,4-diarylated pyridines $\uparrow$}

\author{
Sankar K. Guchhait, ${ }^{* a}$ Neha Hura, ${ }^{a}$ Kanchan Sinha ${ }^{\mathrm{b}}$ and Dulal Panda ${ }^{\mathrm{b}}$ \\ An efficient route for the synthesis of penta-substituted/functionalized-3,4-diarylated pyridines, \\ biologically important templates, via pyridine C3-arylation of nicotinic acids has been developed. The \\ poly-substituted nicotinic acid precursors were prepared by an established multicomponent \\ condensation approach. This route shows an excellent opportunity for introducing versatile (hetero)aryls \\ and other substituents/functionalities into the pyridine ring. Several of the synthesized compounds \\ exhibited significant anti-proliferative properties.
}

\section{Introduction}

Poly-substituted/functionalized pyridines are omnipresent in natural products, bioactive compounds, and functional materials. ${ }^{1}$ In particular, aryl or poly-aryl substituted pyridine derivatives have attracted considerable synthesis attention because they are often found as a valuable structural motif in a wide range of pharmaceutically active compounds (Fig. 1). Etoricoxib that contains the 2,3-diarylpyridine motif is a COX-2 selective inhibitor. $^{2,3}$ 2,4,6-Triarylated pyridine compounds exhibit topoisomerase inhibition (Fig. 1). ${ }^{4}$ Perampanel is a 1,3,5-triarylated pyridine derivative that acts as an AMPA-receptor antagonist. ${ }^{5}$ 5,6-

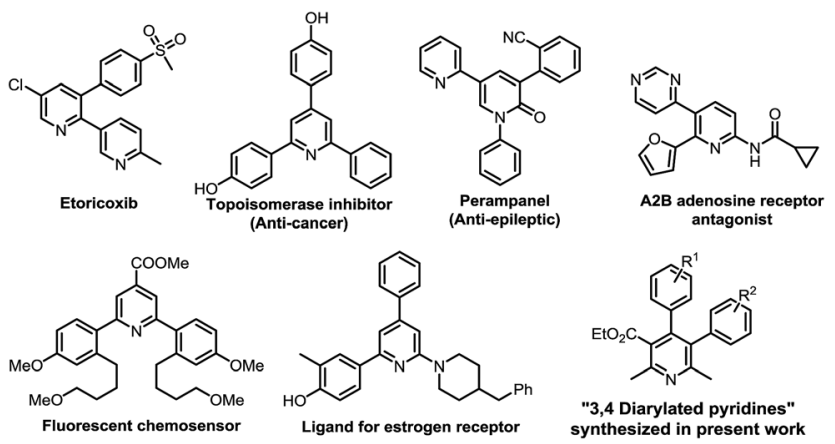

Fig. 1 Selected bioactive agents containing the poly-arylated pyridine structure.

${ }^{a}$ Department of Medicinal Chemistry, National Institute of Pharmaceutical Education and Research (NIPER), S. A. S. Nagar, Mohali-160062, India. E-mail: skguchhait@ niper.ac.in; Fax: +91 172 2214692; Tel: +91 1722214683

${ }^{b}$ Department of Biosciences and Bioengineering, Indian Institute of Technology Bombay, Powai, Mumbai 400076, India

$\dagger$ Electronic supplementary information (ESI) available. See DOI: 10.1039/c6ra28299g
Diheteroarylpyridin-2-carboxamide derivative is a selective A2B adenosine receptor antagonist. ${ }^{6}$ 2-Amino-4,6-diarylpyridines have been found to be potent ligands for estrogen receptor. ${ }^{7}$ There are numerous bioactive natural products that contain polyarylated pyridines. Nosiheptide ${ }^{8}$ has 1,5,6-triarylated 3-hydroxypyridine and promothiocin $\mathrm{A}^{9}$ has 5,6-diarylated pyridine-1-carboxamide and both are potent antibiotics. 2,6-Diarylpyridine-carboxylic ester is useful as a fluorescent chemosensor. ${ }^{10}$

In recent times, as a part of current momentum of exploring new useful organic functional/bioactive materials, the synthesis of compounds that possess not only important heterocyclic scaffold(s) but also structurally resembles in whole molecular skeleton to drugs/bioactive agents has become a valuable research area to organic chemists. ${ }^{\mathbf{1 1}}$ In this direction, late stage functionalization of drugs has also attracted significant attention. ${ }^{12}$ The incorporation of trimethoxyphenyl moiety in target organic compounds is valuable, since it plays role as an important pharmacophoric motif in exhibiting biological properties ${ }^{\mathbf{1 3}}$ and is present in natural products. ${ }^{14}$ Keeping these aspects in mind, we were interested ${ }^{15}$ in the synthesis of 3,4-diarylated pyridines which contain structural features, 3,4,5-trimethoxyphenyl as an aryl motif, and resemblance to a nifedipine drug ${ }^{\mathbf{1 6}}$ and biologically important nicotinic acid. ${ }^{\mathbf{1 7}}$

Over the years, numerous synthetic strategies have been developed to access poly-functionalized pyridines. ${ }^{18,19}$ They involve majorly a pyridine-forming step of appropriately functionalized precursors ${ }^{20}$ or the sequential introduction of substituents on the preformed pyridine ring. ${ }^{21}$ The first strategy is limited to accessibility of particularly-functionalized precursor, while the later strategy includes mainly aromatic substitutions, ${ }^{22}$ direct metallation, or metal halogen-exchange reactions. ${ }^{23}$ Here we report a novel route for synthesis of allsubstituted/functionalized pyridines containing 3,4-diaryl moieties via one-pot pyridine-3-arylation of nicotinic acid skeleton that is easily accessible by an established multicomponent reaction approach. 


\section{Results and discussion}

At the outset, we investigated for preparation of nicotinic acid precursor (5a) by a Hantzsch multicomponent condensation of aryl aldehyde (1a), ethyl acetoacetate (2a) and ammonium acetate to construct dihydropyridine (3a), its dehydrogenative aromatization to product $\mathbf{4 a}$, and mono-hydrolysis of di-ester (Scheme 1). Hantzsch reaction by a reported method was good yielding. ${ }^{24}$ Dehydrogenative aromatization reactions utilizing different conditions ${ }^{25}$ were performed (see ESI, Table $1 \dagger)$. Highest yield was obtained using $\mathrm{Mn}(\mathrm{OAc})_{3}$ and $\mathrm{AcOH}$ at room temperature. Initially, the mono-hydrolysis of pyridine3,5-diesters 4 a was found to be difficult. Both the ester functionalities underwent simultaneously the hydrolysis. A survey of various conditions revealed that 2 equiv. $\mathrm{NaOH}$ in $2 \mathrm{~mL}$ of EtOH- $\mathrm{H}_{2} \mathrm{O}$ (3:1) was most effective for promoting monohydrolysis to produce nicotinic acid 5a (see ESI, Table $2 \dagger$ ).

Next, we investigated to explore decarboxylative arylation of nicotinic acid $\mathbf{5 a}$, with the goal of finding a convenient method for synthesis of penta-substituted/functionalized pyridines containing 3,4-diaryl moieties (Route A, Scheme 2). Several methods of decarboxylative arylation ${ }^{26}$ known for other scaffolds were investigated for the reaction of compound 5a. The desired product was obtained, but the yield more than $8 \%$ could not be accomplished by the methods as well as variation in reported conditions. We envisaged that protodecarboxylation, ${ }^{27}$ and subsequent $\mathrm{C} 3-\mathrm{H}$ bond arylation could afford C3-arylated pyridine (Route B, Scheme 2). The protodecarboxylation of compound 5a underwent via Ag-carboxylate on treatment with AgOAc (1 equiv.), $\mathrm{K}_{2} \mathrm{CO}_{3}$ (30 mol\%) in DMA (anhyd.) at $140{ }^{\circ} \mathrm{C}$ for $12 \mathrm{~h}$ and the product $6 \mathrm{a}$ was obtained in $80 \%$ yield. Next, the $\mathrm{C} 3-\mathrm{H}$ arylation reactions of compound $\mathbf{6 a}$ following the reported pyridine-arylation methods were performed. Ye

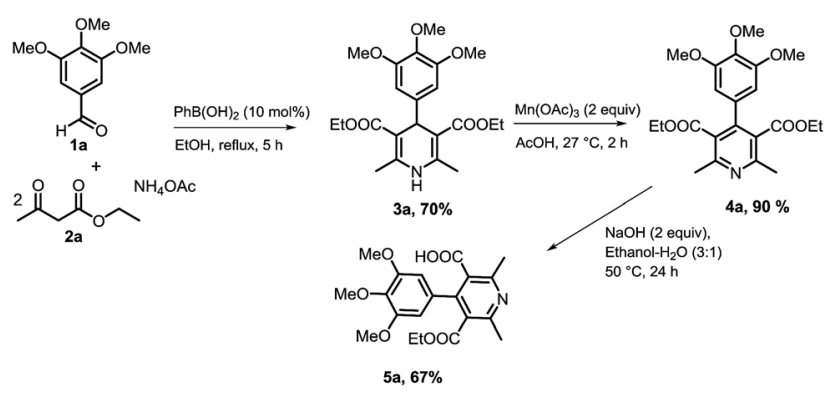

Scheme 1 Synthesis of nicotinic acid derivative 5a.

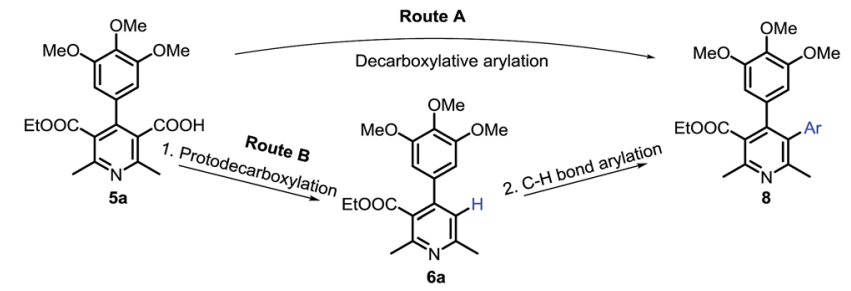

Scheme 2 Strategies towards synthesis of polysubstituted 3,4-diarylated pyridines.

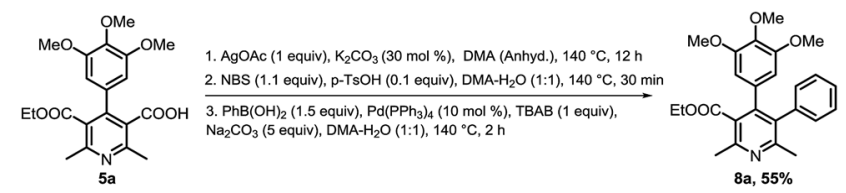

Scheme 3 Optimized reaction condition for one-pot protodecarboxylation-bromination-Suzuki coupling.

developed a Pd-catalyzed C3-selective arylation of unsubstituted pyridine with bromoarenes. ${ }^{28}$ Carrying out C3-arylation of substituted/functionalized pyridine $\mathbf{6 a}$ with bromo- or iodotoluene using Ye's conditions provided 3-arylated pyridine (8a) in poor yields ( $8 \%$ and $10 \%$ yields, respectively). A Pd-catalyzed 3arylation of pyridine with aryl tosylates was reported by Dai. ${ }^{29}$ This methodology did not promote the reaction of compound 6a with $p$-tolyl tosylate. We explored previously a Pd-catalyzed regioselective $\mathrm{C} 6-\mathrm{H}$ arylation of 3-aminoimidazo[1,2-a]pyrazine that underwent via concerted metalation-deprotonation process. $^{30}$ This method promoted the C3-H arylation of pyridine derivative $\mathbf{6 a}$, but the method as well as the variation of its conditions could not improve the product's yield more than $20 \%$. Majorly, the substrate remained intact. The poor conversion and yields in the C3-arylation of pyridine $\mathbf{6 a}$ by reported methods might be due to significant steric hindrance by 4-aryl moiety to inhibit the substrate to undergo the C3-palladation with arylpalladium complex. With aim of obtaining the C-3

Table 1 Evaluation of reagents and conditions ${ }^{a}$

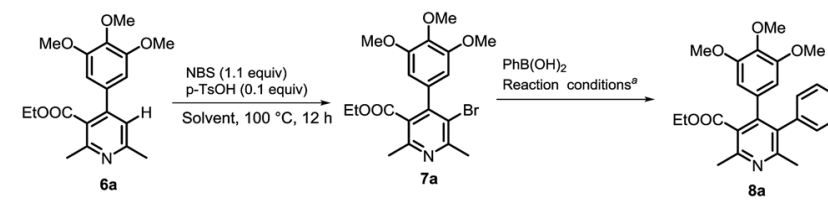

\begin{tabular}{lcc}
\hline Entry & Variable & Yield $^{b}$ \\
\hline Catalyst (10 $\mathbf{~ m o l \% )}, \mathbf{N a}_{2} \mathbf{C O}_{\mathbf{3}}(\mathbf{5}$ equiv. $), \mathbf{T B A B}(\mathbf{1}$ equiv. $), \mathbf{D M A}-\mathbf{H}_{\mathbf{2}} \mathbf{O}(\mathbf{1}: \mathbf{1})$ \\
$\mathbf{P d}\left(\mathrm{PPh}_{3}\right)_{4}$ & 70 \\
2 & $\mathrm{Pd}(\mathrm{dppf}) \mathrm{Cl}_{2}$ & 55 \\
3 & $\mathrm{Pd}\left(\mathrm{PPh}_{3}\right)_{2} \mathrm{Cl}_{2}$ & $\mathrm{NR}$ \\
$4^{c}$ & $\mathrm{Pd}(\mathrm{OAc})_{2}+\mathrm{PPh}_{3}$ & $\mathrm{NR}$ \\
$5^{d}$ & $\mathrm{Pd}\left(\mathrm{PPh}_{3}\right)_{4}$ & 40
\end{tabular}

$\operatorname{Pd}\left(\mathrm{PPh}_{3}\right)_{4}(10 \mathrm{~mol} \%)$, base (5 equiv.), TBAB (1 equiv.), DMA- $\mathrm{H}_{2} \mathrm{O}(1: 1)$

$\begin{array}{lll}6 & \mathrm{~K}_{2} \mathrm{CO}_{3} & 60 \\ 7 & \mathrm{Cs}_{2} \mathrm{CO}_{3} & 55 \\ 8 & \mathrm{~K}_{3} \mathrm{PO}_{4} & 40 \\ 9^{e} & \mathrm{Na}_{2} \mathrm{CO}_{3} & 45\end{array}$

$\mathrm{Pd}(\mathrm{PPh} 3)_{4}$ (10 mol\%), $\mathrm{Na}_{2} \mathrm{CO}_{3}$ (5 equiv.), TBAB (1 equiv.), solvent $10 \quad$ EtOH- $\mathrm{H}_{2} \mathrm{O}(1: 1) \quad 60$ 11 DMA 55 $12^{f} \quad$ EtOH

${ }^{a}$ Reagents and conditions: $\mathrm{PhB}(\mathrm{OH})_{2}$ (1.5 equiv.), $100{ }^{\circ} \mathrm{C}, \mathrm{Ar}, 2 \mathrm{~h}$; $0.5 \mathrm{mmol}$ scale. ${ }^{b}$ Yield for maximum conversion in optimum time. ${ }^{c} \mathrm{PPh}_{3}$ (40 mol\%). ${ }^{d} \mathrm{Pd}\left(\mathrm{PPh}_{3}\right)_{4} \quad(5 \mathrm{~mol} \%) .{ }^{e} \mathrm{Na}_{2} \mathrm{CO}_{3} \quad$ (2.5 equiv.).

${ }^{f}$ Reaction temperature: $80^{\circ} \mathrm{C}$. 
arylated product, we then investigated for an alternate approach involving protodecarboxylation, C3-bromination and Suzuki coupling sequentially (Scheme 3). C3-Bromination ${ }^{31}$ of pyridine 6a leading to product $7 \mathbf{a}$ was found to be nearly quantitative (95\%). Therefore, we were interested to explore one-pot conditions for bromination-Suzuki coupling. $\mathrm{A} \operatorname{Pd}(\mathrm{OAc})_{2}$-catalyzed method reported by $\mathrm{Liu}^{32}$ for Suzuki reaction of $N$-heteroaryl halides was followed in the one-pot reaction of pyridine $\mathbf{6 a}$ with phenylboronic acid, however, the desired product was obtained in $25 \%$ yield only. Christakakou' $\mathrm{s}^{33}$ reaction conditions yielded the product 8 a in $40 \%$ yield only. Gratifyingly, one-pot bromination and Suzuki coupling of pyridine $\mathbf{6 a}$ with phenylboronic acid using $\mathrm{Pd}\left(\mathrm{PPh}_{3}\right)_{4}$ catalyst, $\mathrm{Na}_{2} \mathrm{CO}_{3}, \mathrm{TBAB}$ in DMA- $\mathrm{H}_{2} \mathrm{O}$ provided 3,4-diaryl-pyridine $\mathbf{8 a}$ in $70 \%$ yield (Table 1, entry 1 ). However, further variation in conditions did not improve yield of the product. Incomplete conversion and inferior yield (15\%) were obtained for the reaction carried out in the presence of open air, indicating requirement of non-aerobic conditions for $\operatorname{Pd}(0)$-catalysis. The reaction without TBAB resulted in reduced yield $(40 \%)$. Other palladium sources were evaluated (Table 1, entries 2-4). $\operatorname{Pd}\left(\mathrm{PPh}_{3}\right)_{4}$ was found to be best. Reducing the Pdcatalyst loading (entry 5) was not beneficial. Among various bases investigated, $\mathrm{Na}_{2} \mathrm{CO}_{3}$ provided best result (entries 6-8). Decreasing the equivalence of sodium carbonate below to 5 equiv. resulted in reduced yield (Table 1, entry 9). DMA or EtOH was found effective solvent for bromination, however, they were inferior for promoting the Suzuki coupling in one-pot. Among various solvents and their mixture with water, DMA- $\mathrm{H}_{2} \mathrm{O}$ was found to be most effective for promoting bromination-arylation in one-pot (entries 10-12).

We then investigated protodecarboxylation of nicotinic acid 5a, bromination and Suzuki coupling sequentially in one-pot. Amazingly, it worked well in one-pot with overall yield of 55\% (Scheme 3). The one-pot multi-reactions synthesis of a target molecule is considered as a useful approach in synthetic organic chemistry. ${ }^{34}$ The present work illustrates an important example of one-pot three-reaction process.

With the optimized procedure, we next investigated to explore its substrate scope and synthesize various substituted pyridines. We were pleased to find that the method was found to be flexible in introducing into pyridine at $\mathrm{C} 3$ a variety of (hetero) aryls (Scheme 4). Aryls containing electron-withdrawing or donating functionalities were incorporated. The biologically relevant (hetero)aryl motifs were also introduced easily at C3position of pyridine derivatives. In the established route, difficulty for pyridine $\mathrm{C} 3$-arylation due to steric hindrance by presence of a multi-substituted aryl (3,4,5-trimethoxyphenyl) at C4position was circumvented.

On survey of literature, we found that Simoni demonstrated an attractive profile of cytotoxicity and apoptosis-inducing activity of 2-(3,4,5-trimethoxyphenyl)-3-(3-hydroxy-4-methoxyphenyl)-pyridine (compound A, Fig. 2). ${ }^{35}$ Zheng documented that 2-(3hydroxy-4-methoxyphenyl)-6-(3,4,5-trimethoxyphenyl)-pyridine (B) inhibited cell survival and growth, comparable to a clinical agent, combretastatin A-4 (CA-4). ${ }^{36}$ These representative classes of compounds reveal that 3,4-diarylated pyridine derivatives
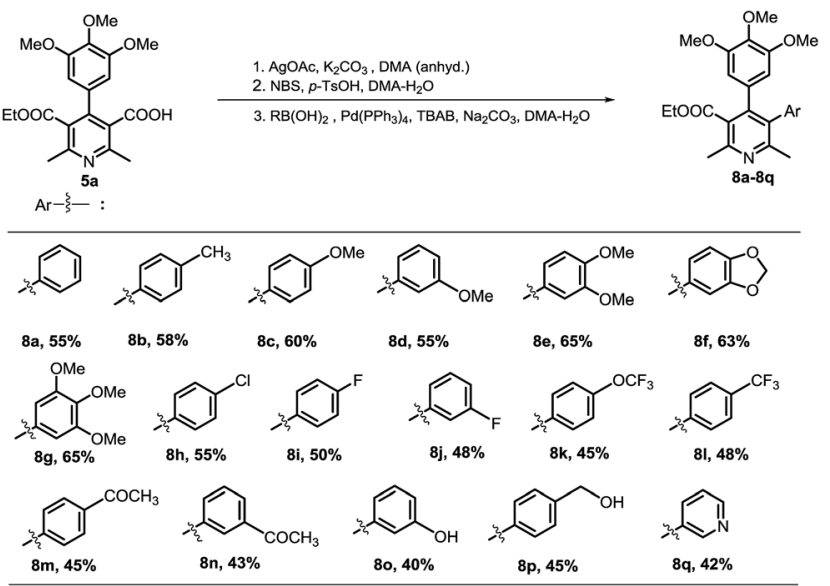

Scheme 4 Substrate scope. ${ }^{a, b}$ asubstrates, reagents and conditions: $5 a(1 \mathrm{mmol}), \mathrm{AgOAc}\left(1\right.$ equiv.), $\mathrm{K}_{2} \mathrm{CO}_{3}$ (30 mol\%), DMA (anhyd., $2 \mathrm{~mL}$ ), $12 \mathrm{~h}$; then, NBS (1.1 equiv.), $p$ - TsOH (0.1 equiv.), DMA- $\mathrm{H}_{2} \mathrm{O}(1: 1,4 \mathrm{~mL})$, 30 min; then, $\mathrm{RB}(\mathrm{OH})_{2}$ (1.5 equiv.), $\mathrm{Pd}\left(\mathrm{PPh}_{3}\right)_{4}$ (10 mol\%), TBAB (1 equiv.), $\mathrm{Na}_{2} \mathrm{CO}_{3}$ (5 equiv.), $140{ }^{\circ} \mathrm{C}, 1-12 \mathrm{~h}$; ${ }^{b}$ yield for maximum conversion in optimum time.

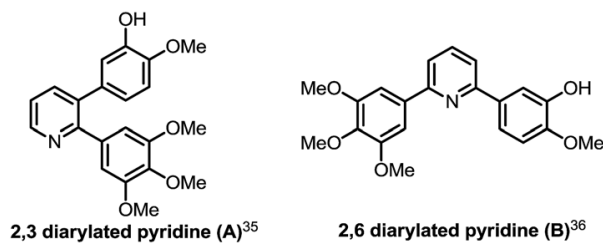

Fig. 2 Reported diarylated pyridines with potent anticancer activity.

synthesized in the present work have potential of exhibiting versatile bioactivities especially the antiproliferative properties.

We extended our developed method applicable to the synthesis of 3,4-diarylated pyridines with relevant substitutions that are present in CA-4 and the diarylated pyridines synthesized and bio-evaluated by Simoni and Zhang.

Ethyl 5-(3-hydroxy-4-methoxyphenyl)-2,6-dimethyl-4-(3,4,5trimethoxyphenyl)nicotinate $(\mathbf{8 r})$ was prepared via an approach (Scheme 5) involving C3-bromination of precursor $\mathbf{6 a}$, boronation $^{37}$ of $7 \mathrm{a}$ with bispinacolatodiborane, and Suzuki coupling with 5-bromo-2-methoxyphenol. This prompted us to synthesize compound 8t with switch in aryl substitutions of

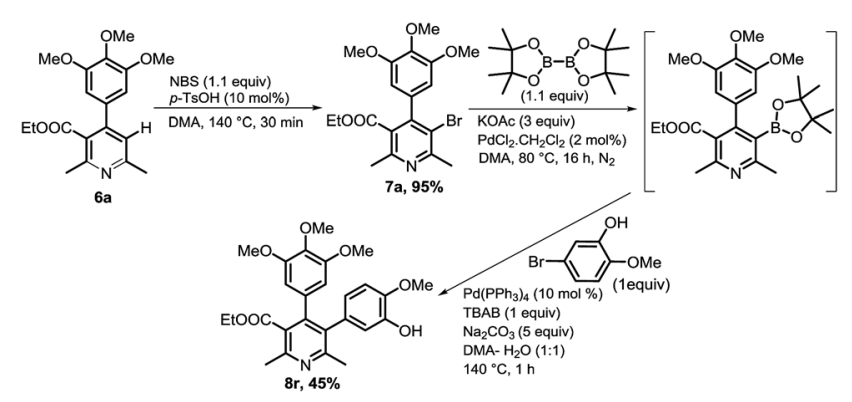

Scheme 5 Synthesis of ethyl 5-(3-hydroxy-4-methoxyphenyl)-2,6dimethyl-4-(3,4,5-trimethoxyphenyl)nicotinate (8r). 


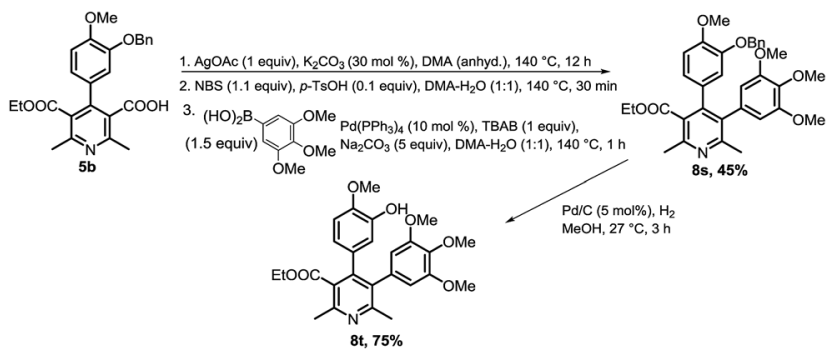

Scheme 6 Synthesis of ethyl 4-(3-hydroxy-4-methoxyphenyl)-2,6dimethyl-5-(3,4,5-trimethoxyphenyl)nicotinate (8t)

compound 8r. We began the preparation of compound $8 \mathbf{t}$ via similar strategy, utilizing 5-(ethoxycarbonyl)-4-(3-hydroxy-4methoxyphenyl)-2,6-dimethylnicotinic acid as the precursor, but bromination did not take place. We anticipated that bromination was problematic due to presence of free hydroxyl group in the precursor. So, benzylation of the hydroxyl group was done to circumvent this problem, and we carried out the synthesis of compound 8t utilizing benzyloxy-aryl derivative of nicotinic acid (5b). One-pot protodecarboxylation-bromination-Suzuki coupling was done to afford compound 8s, which on debenzylation resulted in compound $\mathbf{8 t}$ (Scheme 6).

A set of significantly varied 3,4-diarylated pyridine derivatives (8a-8t) were prepared in the developed approach. Purity (HPLC) of all the compounds were found to be $>95 \%$. Next antiproliferative activity was tested. All compounds were screened for $\%$ inhibition of cell proliferation in HeLa cells as a representative cancer cell line at $5 \mu \mathrm{M}$ concentration (see ESI, Table $3 \dagger$ ). Several of them were found to exhibit considerable antiproliferative activity. Four most potent compounds (pyridines $\mathbf{8 b}, \mathbf{8 f}, \mathbf{8 j}, \mathbf{8 p}$ ) were further evaluated for in vitro tubulin polymerization inhibition (see ESI, Fig. $1 \dagger$ ). The non-activity revealed that the antitubulin may not be the pathway for exhibiting cytotoxicity.

\section{Conclusions}

In conclusion, we have developed an efficient route for synthesis of all-substituted/functionalized pyridines containing 3,4-diaryl moieties. It involves the preparation of nicotinic acids via multicomponent condensation and their pyridine C3-arylation via onepot protodecarboxylation-bromination-Suzuki coupling. A wide range of (hetero)aryls, including especially the biologically important aryl-motifs can be easily introduced in this route. The structural features of the products, structural resemblance to pharmacologically important agent as well as drug and the presence of a biologically important motif 3,4,5-trimethoxyphenyl as one aryl ring, indicate that these functionalized 3,4-diarylpyridines have potential application in finding of versatile bioactive agents. Several of the synthesized compounds were found to exhibit significant anti-proliferative activity.

\section{Experimental}

\section{Chemistry}

General information. ATR \& IR (KBr) Microscope spectrometer was used to record Infrared (IR (KBr)) spectra. ${ }^{1} \mathrm{H}$ NMR spectra were taken on a $400 \mathrm{MHz}$ spectrometer. Data were reported in sequence of chemical shifts in ppm from tetramethylsilane as an internal standard in $\mathrm{CDCl}_{3} / \mathrm{CD}_{3} \mathrm{OD}$, integration, multiplicity $(\mathrm{s}=$ singlet, $\mathrm{d}=$ doublet, $\mathrm{t}=$ triplet, $\mathrm{q}=$ quartet, $\mathrm{m}$ $=$ multiplet, $\mathrm{dt}=$ doublet of triplet, $\mathrm{dd}=$ doublet of doublet, $\mathrm{br}$ $=$ broad), and coupling constants (Hz). ${ }^{13} \mathrm{C}$ NMR spectra were recorded on a $100 \mathrm{MHz}$ spectrometer with protons-decoupling. High-resolution mass spectra (HRMS) were recorded on a highresolution LCMS/MS instrument with "QTOF" mass analyzer. Thin-layer chromatography (TLC) analysis was done using commercially received pre-coated TLC plates (silica gel 60 GF434, $0.43 \mathrm{~mm}$ ). Column chromatography silica gel 100-200 (silica gel 100-200 mesh, neutral, spherical) was used for purification of products. The starting materials and solvents were used as received from commercial sources without further purification.

Representative experimental procedure for the synthesis of diethyl 2,6-dimethyl-4-(3,4,5-trimethoxyphenyl)-1,4-dihydropyridine-3,5-dicarboxylate (Scheme 1, 3a). ${ }^{24}$ 3,4,5-Trimethoxybenzaldehyde 1a (1 mmol, $196 \mathrm{mg})$, ethyl acetoacetate $2 \mathrm{a}$ ( $2 \mathrm{mmol}, 260 \mathrm{mg}, 2$ eq.), $\mathrm{NH}_{4} \mathrm{OAc}(2 \mathrm{mmol}, 154 \mathrm{mg}, 2$ eq.), and $\mathrm{PhB}(\mathrm{OH})_{2}(0.1 \mathrm{mmol}, 12 \mathrm{mg}, 0.1$ eq. $)$ were taken in a round bottom flask and refluxed in EtOH $(2 \mathrm{~mL})$ for $5 \mathrm{~h}$. The reaction mixture was poured into ice cold $\mathrm{H}_{2} \mathrm{O}$. It was then extracted with EtOAc $(2 \times 10 \mathrm{~mL})$. The organic solution was washed with brine, dried over $\mathrm{Na}_{2} \mathrm{SO}_{4}$, and concentrated under vacuum. The crude product was purified by recrystallization from $\mathrm{EtOH}$, which provided 1,4-dihydropyridine $3 \mathbf{a}$ in $70 \%$ yield.

Representative experimental procedure for the synthesis of diethyl 2,6-dimethyl-4-(3,4,5-trimethoxyphenyl)pyridine-3,5dicarboxylate (Scheme 1, 4a). ${ }^{25 a}$ Diethyl 1,4-dihydro-2,6dimethyl-4-(3,4,5-trimethoxyphenyl)pyridine-3,5-dicarboxylate

(3a) (1 mmol, $253 \mathrm{mg}$ ), manganese triacetate ( $2 \mathrm{mmol}, 536 \mathrm{mg}, 2$ eq.) and acetic acid $(5 \mathrm{~mL})$ were taken in a round bottom flask. The reaction mixture was stirred at room temperature till the completion of reaction. Manganese diacetate was filtered through celite bed and the mixture was poured into ice-cold water. Ice-cold aqueous $\mathrm{NaHCO}_{3}$ solution was added dropwise to neutralize the mixture. It was then extracted with dichloromethane $(2 \times 10 \mathrm{~mL})$ and dried over anhydrous sodium sulfate. The solution was concentrated under reduced pressure. The resulting crude mixture on crystallisation from ethanol provided pure product $4 \mathrm{a}$ in $90 \%$ yield.

Representative experimental procedure for the synthesis of 2,6-dimethyl-5-(ethoxycarbonyl)-4-(3,4,5-trimethoxyphenyl)nicotinic acid (Scheme 1, 5a). Aqueous solution of $\mathrm{KOH}(2 \mathrm{mmol}$, $112 \mathrm{mg}, 2$ eq.) in $0.5 \mathrm{~mL}$ water was added to a solution of compound $4 \mathrm{a}(1 \mathrm{mmol}, 417 \mathrm{mg})$ in ethanol $(1.5 \mathrm{~mL})$. The mixture was refluxed $\left(80^{\circ} \mathrm{C}\right)$ till completion of the reaction as indicated by TLC $(7 \mathrm{~h})$. The solvent was evaporated and the crude mass obtained was redissolved in methanol. It was then neutralised to $\mathrm{pH} 7$ with dropwise addition of ice-cold methanolic HCl. The organic solution was concentrated under reduced pressure. The column chromatographic purification of crude mass on silica gel eluting with MeOH-EtOAc (1:9) provided the product $5 \mathrm{a}$ in $67 \%$ yield. 
Representative experimental procedure for synthesis of 2,6dimethyl-3-(ethoxycarbonyl)-5-phenyl-4-(3,4,5-trimethoxyphenyl)pyridine (Scheme 4, entry 1) (8a). Monoacid 5a (1 mmol, 389 $\mathrm{mg}$ ), AgOAc ( $1 \mathrm{mmol}, 167 \mathrm{mg}, 1$ eq.) and $\mathrm{K}_{2} \mathrm{CO}_{3}(0.30 \mathrm{mmol}$, $41.5 \mathrm{mg}, 0.3 \mathrm{eq}$.) were taken under nitrogen in an oven-dried sealed tube equipped with a rubber septum and magnetic bar. DMA (anhyd., $2 \mathrm{~mL}$ ) was added under nitrogen. The tube was then sealed. The mixture was stirred at $140{ }^{\circ} \mathrm{C}$. Upon completion of reaction as indicated by TLC $(12 \mathrm{~h}), \mathrm{N}$-bromosuccinimide (1.1 mmol, $196 \mathrm{mg}, 1.1$ equiv.), $p$-TsOH (0.1 mmol, $17 \mathrm{mg}, 0.1$ eq.) and water $(2 \mathrm{~mL})$ were added in the reaction tube. After completion of the bromination reaction after $30 \mathrm{~min}$, sodium carbonate ( $5 \mathrm{mmol}, 530 \mathrm{mg}, 5$ eq.), tetrabutyl ammonium bromide (1 mmol, $322 \mathrm{mg}, 1$ eq.), phenylboronic acid (1.5 mmol, $182 \mathrm{mg}, 1.5$ eq.) and $\mathrm{Pd}\left(\mathrm{PPh}_{3}\right)_{4}(0.1 \mathrm{mmol}, 115 \mathrm{mg}$, 0.1 eq.) were added to the reaction tube under nitrogen. The tube was then sealed. The mixture was stirred at $140{ }^{\circ} \mathrm{C}$ till the completion of reaction monitored by TLC $(2 \mathrm{~h})$. Then, resultant mixture was allowed to cool to room temperature, diluted with ethyl acetate $(20 \mathrm{~mL})$. The organic layer was washed with aqueous solution of ammonia $(3 \times 5 \mathrm{~mL})$ and brine $(5 \mathrm{~mL})$, dried over $\mathrm{Na}_{2} \mathrm{SO}_{4}$ and the organic layer was concentrated under reduced pressure. The column chromatographic purification of crude mass on silica gel eluting with EtOAc-hexane $(1: 3)$ provided 2,6-dimethyl-3-(ethoxycarbonyl)-5-phenyl-4-(3,4,5trimethoxyphenyl)pyridine $8 \mathrm{a}$ in $55 \%$ overall yield.

Products (8b-8t) were also prepared following this representative procedure.

Diethyl 2,6-dimethyl-4-(3,4,5-trimethoxyphenyl)-1,4-dihydropyridine-3,5-dicarboxylate (3a). ${ }^{38}$ Yellow solid; $293 \mathrm{mg}$, 70\%; mp $140{ }^{\circ} \mathrm{C} ;{ }^{1} \mathrm{H} \mathrm{NMR}\left(400 \mathrm{MHz}, \mathrm{CDCl}_{3}\right): \delta 6.52(\mathrm{~s}, 2 \mathrm{H}), 5.77(\mathrm{~s}, 1 \mathrm{NH})$, $4.98(\mathrm{~s}, 1 \mathrm{H}), 4.17-4.08(\mathrm{~m}, 4 \mathrm{H}), 3.79(\mathrm{~s}, 9 \mathrm{H}), 2.34(\mathrm{~s}, 6 \mathrm{H}), 1.25(\mathrm{t}, J$ $=7.1 \mathrm{~Hz}, 6 \mathrm{H}) \mathrm{ppm} ;{ }^{13} \mathrm{C}\left\{{ }^{1} \mathrm{H}\right\} \mathrm{NMR}\left(100 \mathrm{MHz}, \mathrm{CDCl}_{3}\right): \delta 167.7$, 152.6, 143.9, 143.4, 105.0, 103.9, 60.8, 59.8, 55.9, 19.6, 14.4 ppm; IR (KBr): $\nu_{\max } 3354,2987,1650,1232,1123,1006 \mathrm{~cm}^{-1}$.

Diethyl 2,6-dimethyl-4-(3,4,5-trimethoxyphenyl)pyridine-3,5dicarboxylate (4a). White solid; $375 \mathrm{mg}$, 90\%; mp $130{ }^{\circ} \mathrm{C} ;{ }^{1} \mathrm{H}$ NMR (400 MHz, $\left.\mathrm{CDCl}_{3}\right): \delta 6.51(\mathrm{~s}, 2 \mathrm{H}), 4.08(\mathrm{q}, J=7.1 \mathrm{~Hz}, 4 \mathrm{H}), 3.86$ $(\mathrm{s}, 3 \mathrm{H}), 3.83(\mathrm{~s}, 6 \mathrm{H}), 2.60(\mathrm{~s}, 6 \mathrm{H}), 0.99(\mathrm{t}, J=7.1 \mathrm{~Hz}, 6 \mathrm{H}) \mathrm{ppm} ;{ }^{13} \mathrm{C}$ $\left\{{ }^{1} \mathrm{H}\right\}$ NMR (100 MHz, $\left.\mathrm{CDCl}_{3}\right): \delta 168.0,155.4,152.9,145.7,138.1$, 131.9, 126.8, 105.5, 61.5, 60.9, 56.2, 22.8, 13.7 ppm; IR (KBr): $\nu_{\max }$ 2987, 1719, 1585, 1232, 1123, $1006 \mathrm{~cm}^{-1}$; HRMS (ESI) $\mathrm{m} / \mathrm{z}$ : calcd for $\mathrm{C}_{22} \mathrm{H}_{28} \mathrm{NO}_{7}[\mathrm{M}+\mathrm{H}]^{+}$418.1866, found: 418.1858.

2,6-Dimethyl-5-(ethoxycarbonyl)-4-(3,4,5-trimethoxyphenyl) nicotinic acid (5a). Off white solid; $260 \mathrm{mg}, 67 \%$; mp charred at $225{ }^{\circ} \mathrm{C} ;{ }^{1} \mathrm{H}$ NMR $\left(400 \mathrm{MHz}, \mathrm{CDCl}_{3}\right): \delta 7.03(\mathrm{~s}, 1 \mathrm{H}), 6.58(\mathrm{~s}, 2 \mathrm{H})$, $4.14(\mathrm{q}, J=7.2 \mathrm{~Hz}, 2 \mathrm{H}), 3.88(\mathrm{~s}, 3 \mathrm{H}), 3.87(\mathrm{~s}, 6 \mathrm{H}), 2.60(\mathrm{~s}, 3 \mathrm{H})$, 2.59 (s, 3H), 1.06 (t, $J=7.2 \mathrm{~Hz}, 3 \mathrm{H}) \mathrm{ppm} ;{ }^{13} \mathrm{C}\left\{{ }^{1} \mathrm{H}\right\}$ NMR $(100$ $\left.\mathrm{MHz}, \mathrm{CDCl}_{3}\right): \delta 169.2,158.7,155.0,153.3,148.2,138.2,134.3$, 125.8, 120.8, 105.1, 61.4, 60.9, 56.2, 24.5, 22.8, 13.8 ppm; IR (KBr): $\nu_{\max } 3423,2970,1718,1582,1249,1122,1010 \mathrm{~cm}^{-1}$; HRMS (ESI) $m / z$ : calcd for $\mathrm{C}_{20} \mathrm{H}_{24} \mathrm{NO}_{7}[\mathrm{M}+\mathrm{H}]^{+} 390.1553$, found: 390.1547.

2,6-Dimethyl-3-(ethoxycarbonyl)-5-phenyl-4-(3,4,5-

trimethoxyphenyl)pyridine (8a). White solid; $232 \mathrm{mg}, 55 \%$; $\mathrm{mp}$ $120{ }^{\circ} \mathrm{C} ;{ }^{1} \mathrm{H}$ NMR $\left(400 \mathrm{MHz}, \mathrm{CDCl}_{3}\right): \delta 7.20-7.19(\mathrm{~m}, 5 \mathrm{H}), 6.56(\mathrm{~s}$,
1H), $6.42(\mathrm{~s}, 1 \mathrm{H}), 4.15-4.12(\mathrm{~m}, 2 \mathrm{H}), 3.95(\mathrm{~s}, 3 \mathrm{H}), 3.84(\mathrm{~s}, 3 \mathrm{H})$, $3.59(\mathrm{~s}, 3 \mathrm{H}), 2.57(\mathrm{~s}, 3 \mathrm{H}), 2.26(\mathrm{~s}, 3 \mathrm{H}), 1.03(\mathrm{t}, J=7.1 \mathrm{~Hz}$, $3 \mathrm{H}) \mathrm{ppm} ;{ }^{13} \mathrm{C}\left\{{ }^{1} \mathrm{H}\right\} \mathrm{NMR}\left(100 \mathrm{MHz}, \mathrm{CDCl}_{3}\right): \delta 168.9,157.5,155.1$, 152.3 , 151.7, 148.4, 142.3, 135.7, 133.6, 130.6, 127.9, 127.6, 126.8, 126.3, 122.7, 108.3, 61.2, 61.1, 60.9, 56.1, 24.2, 23.0, 13.7 ppm; IR (KBr): $\nu_{\max } 2978,2935,2850,1726,1586,1485$, $1264,1096 \mathrm{~cm}^{-1}$; HRMS (ESI) $m / z$ : calcd for $\mathrm{C}_{25} \mathrm{H}_{28} \mathrm{NO}_{5}[\mathrm{M}+\mathrm{H}]^{+}$ 422.1967, found: 422.1958 .

2,6-Dimethyl-3-(ethoxycarbonyl)-5-(p-tolyl)-4-(3,4,5-trimethoxyphenyl)pyridine (8b). White solid; $252 \mathrm{mg}, 58 \%$; mp $123{ }^{\circ} \mathrm{C} ;{ }^{1} \mathrm{H}$ NMR (400 MHz, $\left.\mathrm{CDCl}_{3}\right): \delta 7.07(\mathrm{~d}, J=7.1 \mathrm{~Hz}, 2 \mathrm{H}), 7.00$ (d, $J=$ $7.7 \mathrm{~Hz}, 2 \mathrm{H}), 6.54(\mathrm{~s}, 1 \mathrm{H}), 6.44(\mathrm{~s}, 1 \mathrm{H}), 4.15-4.09(\mathrm{~m}, 2 \mathrm{H}), 3.94(\mathrm{~s}$, $3 \mathrm{H}), 3.84(\mathrm{~s}, 3 \mathrm{H}), 3.60(\mathrm{~s}, 3 \mathrm{H}), 2.57(\mathrm{~s}, 3 \mathrm{H}), 2.29(\mathrm{~s}, 3 \mathrm{H}), 2.28(\mathrm{~s}, 3 \mathrm{H})$ $1.02(\mathrm{t}, J=7.1 \mathrm{~Hz}, 3 \mathrm{H}) \mathrm{ppm} ;{ }^{13} \mathrm{C}\left\{{ }^{1} \mathrm{H}\right\}$ NMR (100 MHz, $\left.\mathrm{CDCl}_{3}\right)$ : $\delta 168.9,157.5,155.1,152.1,151.8,148.6,142.2,136.3,133.7,132.6$, 130.5, 128.4, 127.8, 126.3, 122.8, 108.2, 61.1, 61.0, 60.9, 56.1, 24.2, 23.1, 21.2, 13.7 ppm; IR (KBr): $\nu_{\max } 2925,2854,1724,1587,1488$, $1263,1087 \mathrm{~cm}^{-1}$; HRMS (ESI) $m / z$ : calcd for $\mathrm{C}_{26} \mathrm{H}_{30} \mathrm{NO}_{5}[\mathrm{M}+\mathrm{H}]^{+}$ 436.2124, found: 436.2124 .

2,6-Dimethyl-3-(ethoxycarbonyl)-5-(4-methoxyphenyl)-4-(3,4,5trimethoxyphenyl)pyridine (8c). White solid; $271 \mathrm{mg}, 60 \%$; $\mathrm{mp}>$ $200{ }^{\circ} \mathrm{C} ;{ }^{1} \mathrm{H}$ NMR $\left(400 \mathrm{MHz}, \mathrm{CDCl}_{3}\right): \delta 7.12(\mathrm{~d}, J=7.8 \mathrm{~Hz}, 2 \mathrm{H})$, $6.75(\mathrm{~d}, J=8.4 \mathrm{~Hz}, 2 \mathrm{H}), 6.53(\mathrm{~s}, 1 \mathrm{H}), 6.44(\mathrm{~s}, 1 \mathrm{H}), 4.15-4.09(\mathrm{~m}$, $2 \mathrm{H}), 3.94(\mathrm{~s}, 3 \mathrm{H}), 3.84(\mathrm{~s}, 3 \mathrm{H}), 3.77(\mathrm{~s}, 3 \mathrm{H}), 3.58(\mathrm{~s}, 3 \mathrm{H}), 2.58(\mathrm{~s}$, $3 \mathrm{H}), 2.30(\mathrm{~s}, 3 \mathrm{H}), 1.02(\mathrm{t}, J=7.1 \mathrm{~Hz}, 3 \mathrm{H}) \mathrm{ppm} ;{ }^{13} \mathrm{C}\left\{{ }^{1} \mathrm{H}\right\} \mathrm{NMR}(100$ $\left.\mathrm{MHz}, \mathrm{CDCl}_{3}\right): \delta 168.9,158.3,157.6,155.1,152.1,151.8,148.7$, 142.3, 133.7, 131.7, 127.9, 127.4, 126.4, 122.7, 113.1, 108.2, 61.2, 61.1, 60.9, 56.1, 55.1, 24.3, 23.1, 13.7 ppm; IR (KBr): $\nu_{\max } 2930$, 2850, 1725, 1586, 1488, 1262, 1245, $1096 \mathrm{~cm}^{-1}$; HRMS (ESI) m/z: calcd for $\mathrm{C}_{26} \mathrm{H}_{30} \mathrm{NO}_{6}[\mathrm{M}+\mathrm{H}]^{+}$452.2073, found: 452.2068.

2,6-Dimethyl-3-(ethoxycarbonyl)-5-(3-methoxyphenyl)-4-(3,4,5trimethoxyphenyl)pyridine (8d). White solid; $248 \mathrm{mg}, 55 \%$; $\mathrm{mp}$ $116{ }^{\circ} \mathrm{C} ;{ }^{1} \mathrm{H}$ NMR $\left(400 \mathrm{MHz}, \mathrm{CDCl}_{3}\right): \delta 7.13(\mathrm{dd}, J=7.8 \mathrm{~Hz}, J=$ $7.7 \mathrm{~Hz}, 1 \mathrm{H}), 6.84-6.78(\mathrm{~m}, 2 \mathrm{H}), 6.74-6.71(\mathrm{~m}, 1 \mathrm{H}), 6.55(\mathrm{~s}, 1 \mathrm{H})$, $6.46(\mathrm{~s}, 1 \mathrm{H}), 4.15-4.12(\mathrm{~m}, 2 \mathrm{H}), 3.95(\mathrm{~s}, 3 \mathrm{H}), 3.84(\mathrm{~s}, 3 \mathrm{H}), 3.65(\mathrm{~s}$, $3 \mathrm{H}), 3.63(\mathrm{~s}, 3 \mathrm{H}), 2.57(\mathrm{~s}, 3 \mathrm{H}), 2.29(\mathrm{~s}, 3 \mathrm{H}), 1.03(\mathrm{t}, J=7.1 \mathrm{~Hz}$, $3 \mathrm{H}) \mathrm{ppm} ;{ }^{13} \mathrm{C}\left\{{ }^{1} \mathrm{H}\right\}$ NMR $\left(100 \mathrm{MHz}, \mathrm{CDCl}_{3}\right): \delta$ 168.9, 158.9, 157.6, 154.9, 152.3, 151.7, 148.5, 142.3, 137.0, 133.5, 128.5, 127.7, 126.3, 123.3, 122.6, 115.9, 112.9, 108.2, 61.2, 61.1, 61.0, 56.1, 55.1, 24.2, 22.9, 13.8 ppm; IR (KBr): $\nu_{\max } 2927,2852,1724,1586,1462,1274$, $1259,1088 \mathrm{~cm}^{-1}$; HRMS (ESI) $m / z$ : calcd for $\mathrm{C}_{26} \mathrm{H}_{30} \mathrm{NO}_{6}[\mathrm{M}+\mathrm{H}]^{+}$ 452.2073, found: 452.2068 .

5-(3,4-Dimethoxyphenyl)-2,6-dimethyl-3-(ethoxycarbonyl)4-(3,4,5-trimethoxyphenyl)pyridine (8e). Pale yellow solid; $313 \mathrm{mg}, 65 \%$; mp $135{ }^{\circ} \mathrm{C} ;{ }^{1} \mathrm{H}$ NMR (400 MHz, $\mathrm{CDCl}_{3}$ ): $\delta$ 6.84$6.79(\mathrm{~m}, 2 \mathrm{H}), 6.74(\mathrm{~d}, J=8.2 \mathrm{~Hz}, 1 \mathrm{H}), 6.55(\mathrm{~s}, 1 \mathrm{H}), 6.46(\mathrm{~s}, 1 \mathrm{H})$, 4.18-4.11 (m, 2H), $3.95(\mathrm{~s}, 3 \mathrm{H}), 3.85(\mathrm{~s}, 3 \mathrm{H}), 3.84(\mathrm{~s}, 3 \mathrm{H}), 3.67$ $(\mathrm{s}, 3 \mathrm{H}), 3.62(\mathrm{~s}, 3 \mathrm{H}), 2.56(\mathrm{~s}, 3 \mathrm{H}), 2.29(\mathrm{~s}, 3 \mathrm{H}), 1.05(\mathrm{t}, J=$ $7.1 \mathrm{~Hz}, 3 \mathrm{H}) \mathrm{ppm} ;{ }^{13} \mathrm{C}\left\{{ }^{1} \mathrm{H}\right\} \mathrm{NMR}\left(100 \mathrm{MHz}, \mathrm{CDCl}_{3}\right): \delta 168.9$, $157.7,154.8,152.1,151.8,148.7,147.9$, 147.7, 142.3, 133.6, 128.2, 127.5, 126.4, 123.1, 122.5, 114.1, 110.3, 108.3, 61.2, 61.1, 60.9, 56.1, 55.7, 24.3, 22.9, 13.8 ppm; IR (KBr): $\nu_{\max } 2924$, 2853, 1726, 1586, 1463, 1248, $1086 \mathrm{~cm}^{-1}$; HRMS (ESI) $\mathrm{m} / z$ : calcd for $\mathrm{C}_{26} \mathrm{H}_{32} \mathrm{NO}_{7}[\mathrm{M}+\mathrm{H}]^{+} 482.2179$, found: 482.2181 .

5-(Benzo[d][1,3]dioxol-5-yl)-2,6-dimethyl-3-(ethoxycarbonyl)4-(3,4,5-trimethoxyphenyl)pyridine (8f). White solid; $293 \mathrm{mg}$, $63 \%$; mp $115{ }^{\circ} \mathrm{C} ;{ }^{1} \mathrm{H}$ NMR (400 $\left.\mathrm{MHz}, \mathrm{CDCl}_{3}\right): \delta 6.73-6.67(\mathrm{~m}$, 
$3 \mathrm{H}), 6.52(\mathrm{~s}, 1 \mathrm{H}), 6.49(\mathrm{~s}, 1 \mathrm{H}), 5.92(\mathrm{~s}, 2 \mathrm{H}), 4.16-4.09(\mathrm{~m}, 2 \mathrm{H})$, $3.94(\mathrm{~s}, 3 \mathrm{H}), 3.83$ (s, 3H), $3.63(\mathrm{~s}, 3 \mathrm{H}), 2.58(\mathrm{~s}, 3 \mathrm{H}), 2.34(\mathrm{~s}, 3 \mathrm{H})$, $1.02(\mathrm{t}, J=7.1 \mathrm{~Hz}, 3 \mathrm{H}) \mathrm{ppm} ;{ }^{13} \mathrm{C}\left\{{ }^{1} \mathrm{H}\right\}$ NMR (100 MHz, $\left.\mathrm{CDCl}_{3}\right)$ : $\delta$ 168.8, 157.6, 155.3, 152.2, 151.8, 148.6, 146.9, 146.3, 142.2, $133.7,129.4,127.3,126.3,124.3,122.5,111.1,108.1,107.7$, 100.8, 61.2, 61.1, 60.9, 56.1, 24.3, 23.1, 13.7 ppm; IR (KBr): $\nu_{\max }$ 2925, 2851, 1723, 1586, 1481, 1455, 1262, 1233, 1123, 1081 $\mathrm{cm}^{-1}$; HRMS (ESI) $\mathrm{m} / \mathrm{z}$ : calcd for $\mathrm{C}_{26} \mathrm{H}_{28} \mathrm{NO}_{7}[\mathrm{M}+\mathrm{H}]^{+}$466.1866, found: 466.1866 .

2,6-Dimethyl-3-(ethoxycarbonyl)-4,5-bis(3,4,5-trimethoxyphenyl)pyridine (8g). Yellow semisolid; $332 \mathrm{mg}, 65 \%$; ${ }^{1} \mathrm{H}$ NMR $(400 \mathrm{MHz}$, $\left.\mathrm{CDCl}_{3}\right): \delta 6.57(\mathrm{~s}, 1 \mathrm{H}), 6.51(\mathrm{~s}, 2 \mathrm{H}), 6.47(\mathrm{~s}, 1 \mathrm{H}), 4.18-4.12(\mathrm{~m}, 2 \mathrm{H})$, 3.95 (s, 3H), 3.85 (s, 3H), 3.80 (s, 3H), 3.68 (s, 9H), 2.56 (s, 3H), 2.31 (s, 3H), 1.06 (t, $J=7.1 \mathrm{~Hz}, 3 \mathrm{H}) \mathrm{ppm} ;{ }^{13} \mathrm{C}\left\{{ }^{1} \mathrm{H}\right\}$ NMR $(100 \mathrm{MHz}$, $\left.\mathrm{CDCl}_{3}\right): \delta 168.8,157.7,154.6,152.4,152.2,151.6,148.6,142.3,136.8$, 133.4, 131.1, 127.6, 126.4, 122.2, 108.3, 108.2, 61.3, 61.11, 61.09, 60.9, 56.1, 56.0, 24.2, 22.8, 13.9 ppm; IR (KBr): $\nu_{\max } 2931,2852,1726$, 1586, 1464, 1262, 1127, 1103, $1008 \mathrm{~cm}^{-1}$; HRMS (ESI) $\mathrm{m} / \mathrm{z}$ : calcd for $\mathrm{C}_{28} \mathrm{H}_{34} \mathrm{NO}_{8}[\mathrm{M}+\mathrm{H}]^{+}$512.2284, found: 512.2280.

5-(4-Chlorophenyl)-2,6-dimethyl-3-(ethoxycarbonyl)-4-(3,4,5trimethoxyphenyl)pyridine (8h). White solid; $250 \mathrm{mg}, 55 \%$; $\mathrm{mp}$ $>200{ }^{\circ} \mathrm{C} ;{ }^{1} \mathrm{H}$ NMR $\left(400 \mathrm{MHz}, \mathrm{CDCl}_{3}\right): \delta 7.19-7.14(\mathrm{~m}, 4 \mathrm{H}), 6.54$ $(\mathrm{s}, 1 \mathrm{H}), 6.42(\mathrm{~s}, 1 \mathrm{H}), 4.15-4.12(\mathrm{~m}, 2 \mathrm{H}), 3.94(\mathrm{~s}, 3 \mathrm{H}), 3.84(\mathrm{~s}, 3 \mathrm{H})$, $3.59(\mathrm{~s}, 3 \mathrm{H}), 2.58(\mathrm{~s}, 3 \mathrm{H}), 2.31(\mathrm{~s}, 3 \mathrm{H}), 1.03(\mathrm{t}, J=7.2 \mathrm{~Hz}$, 3H) ppm; ${ }^{13} \mathrm{C}\left\{{ }^{1} \mathrm{H}\right\}$ NMR $\left(100 \mathrm{MHz}, \mathrm{CDCl}_{3}\right): \delta 168.7,157.7,155.3$, 152.5 , 151.6, 148.2, 142.3, 134.2, 133.5, 132.7, 132.0, 127.8, 126.41, 126.36, 122.6, 108.3, 61.2, 61.1, 60.9, 56.1, 24.2, 22.9, 13.7 ppm; IR (KBr): $\nu_{\max } 2934,2851,1724,1585,1483,1261$, 1084, 1005, $827 \mathrm{~cm}^{-1}$; HRMS (ESI) $m / z$ : calcd for $\mathrm{C}_{25} \mathrm{H}_{27} \mathrm{NO}_{6}[\mathrm{M}$ $+\mathrm{H}]^{+}$456.1570, found: 456.1588 .

2,6-Dimethyl-3-(ethoxycarbonyl)-5-(4-fluorophenyl)-4-(3,4,5trimethoxyphenyl)pyridine (8i). White solid; $220 \mathrm{mg}, 50 \%$; mp $145{ }^{\circ} \mathrm{C} ;{ }^{1} \mathrm{H}$ NMR $\left(400 \mathrm{MHz}, \mathrm{CDCl}_{3}\right): \delta$ 7.20-7.17 (m, 2H), 6.91 $(\mathrm{dd}, J=8.5 \mathrm{~Hz}, J=8.4 \mathrm{~Hz}, 2 \mathrm{H}), 6.55(\mathrm{~s}, 1 \mathrm{H}), 6.41$ (s, 1H), 4.17$4.10(\mathrm{~m}, 2 \mathrm{H}), 3.95(\mathrm{~s}, 3 \mathrm{H}), 3.84(\mathrm{~s}, 3 \mathrm{H}), 3.59(\mathrm{~s}, 3 \mathrm{H}), 2.58(\mathrm{~s}, 3 \mathrm{H})$, $2.30(\mathrm{~s}, 3 \mathrm{H}), 1.03(\mathrm{t}, J=7.2 \mathrm{~Hz}, 3 \mathrm{H}) \mathrm{ppm} ;{ }^{13} \mathrm{C}\left\{{ }^{1} \mathrm{H}\right\}$ NMR $(100$ $\left.\mathrm{MHz} \mathrm{CDCl}_{3}\right): \delta 168.8,161.7\left(\mathrm{~d}, J_{\mathrm{C}-\mathrm{F}}=244 \mathrm{~Hz}\right), 157.7,155.2$, 152.4, 151.7, 148.3, 142.3, 133.7, $132.3\left(\mathrm{~d}, J_{\mathrm{C}-\mathrm{C}-\mathrm{C}-\mathrm{F}}=8 \mathrm{~Hz}\right)$, $131.6\left(\mathrm{~d}, J_{\mathrm{C}-\mathrm{C}-\mathrm{C}-\mathrm{C}-\mathrm{F}}=4 \mathrm{~Hz}\right), 126.7,126.3,122.6,114.6\left(\mathrm{~d}, J_{\mathrm{C}-\mathrm{C}-\mathrm{F}}\right.$ $=21 \mathrm{~Hz}), 108.3$, 61.2, 61.1, 60.9, 56.1, 24.2, 23.1, 13.8 ppm; IR (KBr): $\nu_{\max }$ 2959, 2928, 2870, 1716, 1586, 1487, 1257, 1093, $1082,1006 \mathrm{~cm}^{-1}$; HRMS (ESI) $m / z$ : calcd for $\mathrm{C}_{25} \mathrm{H}_{27} \mathrm{FNO}_{5}[\mathrm{M}+$ $\mathrm{H}]^{+}$440.1873, found: 440.1860 .

2,6-Dimethyl-3-(ethoxycarbonyl)-5-(3-fluorophenyl)-4-(3,4,5trimethoxyphenyl)pyridine (8j). White solid; $211 \mathrm{mg}, 48 \%$; $\mathrm{mp}$ $114{ }^{\circ} \mathrm{C} ;{ }^{1} \mathrm{H}$ NMR $\left(400 \mathrm{MHz}, \mathrm{CDCl}_{3}\right): \delta 7.18-7.13(\mathrm{~m}, 1 \mathrm{H}), 6.98-$ $6.96(\mathrm{~m}, 1 \mathrm{H}), 6.91-6.86(\mathrm{~m}, 1 \mathrm{H}), 6.54(\mathrm{~s}, 1 \mathrm{H}), 6.44(\mathrm{~s}, 1 \mathrm{H}), 4.15-$ $4.10(\mathrm{~m}, 2 \mathrm{H}), 3.95(\mathrm{~s}, 3 \mathrm{H}), 3.84(\mathrm{~s}, 3 \mathrm{H}), 3.62(\mathrm{~s}, 3 \mathrm{H}), 2.58(\mathrm{~s}, 3 \mathrm{H})$, $2.30(\mathrm{~s}, 3 \mathrm{H}), 1.03(\mathrm{t}, J=7.2 \mathrm{~Hz}, 3 \mathrm{H}) \mathrm{ppm} ;{ }^{13} \mathrm{C}\left\{{ }^{1} \mathrm{H}\right\}$ NMR $(100$ $\left.\mathrm{MHz}, \mathrm{CDCl}_{3}\right): \delta 168.7,162.2\left(\mathrm{~d}, J_{\mathrm{C}-\mathrm{F}}=243 \mathrm{~Hz}\right), 157.8,155.3$, 152.6, 151.6, 148.1, 142.3, 137.9 (d, $\left.J_{\mathrm{C}-\mathrm{C}-\mathrm{C}-\mathrm{F}}=8 \mathrm{~Hz}\right), 133.6$, $129.0\left(\mathrm{~d}, J_{\mathrm{C}-\mathrm{C}-\mathrm{C}-\mathrm{F}}=8 \mathrm{~Hz}\right), 126.6,126.4,126.3,122.4,117.6(\mathrm{~d}$, $\left.J_{\mathrm{C}-\mathrm{C}-\mathrm{F}}=21 \mathrm{~Hz}\right), 113.7\left(\mathrm{~d}, J_{\mathrm{C}-\mathrm{C}-\mathrm{F}}=21 \mathrm{~Hz}\right), 108.3,61.2,61.1,61.0$, 56.1, 24.2, 23.1, 13.8 ppm; IR (KBr): $\nu_{\max }$ 2955, 2927, 2852, 1724, 1584, 1482, 1260, 1129, 1084, $1014 \mathrm{~cm}^{-1}$; HRMS (ESI) m/ $z$ : calcd for $\mathrm{C}_{25} \mathrm{H}_{27} \mathrm{FNO}_{5}[\mathrm{M}+\mathrm{H}]^{+} 440.1873$, found: 440.1869 .
2,6-Dimethyl-3-(ethoxycarbonyl)-5-(4-(trifluoromethoxy)phenyl)4-(3,4,5-trimethoxyphenyl)pyridine (8k). White solid; $227 \mathrm{mg}, 45 \%$; mp $130{ }^{\circ} \mathrm{C} ;{ }^{1} \mathrm{H}$ NMR (400 MHz, $\left.\mathrm{CDCl}_{3}\right): \delta 7.24(\mathrm{~d}, J=7.6 \mathrm{~Hz}, 2 \mathrm{H})$, $7.06(\mathrm{~d}, J=8.1 \mathrm{~Hz}, 2 \mathrm{H}), 6.57(\mathrm{~s}, 1 \mathrm{H}), 6.36(\mathrm{~s}, 1 \mathrm{H}), 4.16-4.11(\mathrm{~m}, 2 \mathrm{H})$, $3.95(\mathrm{~s}, 3 \mathrm{H}), 3.85(\mathrm{~s}, 3 \mathrm{H}), 3.62(\mathrm{~s}, 3 \mathrm{H}), 2.58(\mathrm{~s}, 3 \mathrm{H}), 2.27(\mathrm{~s}, 3 \mathrm{H}), 1.03$ $(\mathrm{t}, J=7.1 \mathrm{~Hz}, 3 \mathrm{H}) \mathrm{ppm} ;{ }^{13} \mathrm{C}\left\{{ }^{1} \mathrm{H}\right\} \mathrm{NMR}\left(100 \mathrm{MHz}, \mathrm{CDCl}_{3}\right): \delta 168.7$, 157.8, 155.3, 152.6, 151.6, 148.0, 142.3, 134.5, 133.6, 132.1, 126.4, 126.3, 122.5, 120.4 (q, $\left.J_{\mathrm{C}-\mathrm{F}}=255 \mathrm{~Hz}\right), 120.1,108.3,61.2,61.1,60.9$, 56.1, 24.1, 23.1, 13.8 ppm; IR (KBr): $\nu_{\max }$ 2935, 2850, 1725, 1586, 1484, 1251, 1161, 1082, $1005 \mathrm{~cm}^{-1}$; HRMS (ESI) $\mathrm{m} / z$ : calcd for $\mathrm{C}_{26} \mathrm{H}_{27} \mathrm{~F}_{3} \mathrm{NO}_{6}[\mathrm{M}+\mathrm{H}]^{+}$506.1790, found: 506.1787.

2,6-Dimethyl-3-(ethoxycarbonyl)-5-(4-(trifluoromethyl)phenyl)4-(3,4,5-trimethoxyphenyl)pyridine (81). White solid; $235 \mathrm{mg}$, $48 \%$; mp $147{ }^{\circ} \mathrm{C} ;{ }^{1} \mathrm{H}$ NMR (400 MHz, $\left.\mathrm{CDCl}_{3}\right): \delta 7.47$ (d, $J=8 \mathrm{~Hz}$, $2 \mathrm{H}), 7.35$ (d, $J=7.3 \mathrm{~Hz}, 2 \mathrm{H}), 6.57(\mathrm{~s}, 1 \mathrm{H}), 6.38(\mathrm{~s}, 1 \mathrm{H}), 4.18-4.12$ $(\mathrm{m}, 2 \mathrm{H}), 3.95(\mathrm{~s}, 3 \mathrm{H}), 3.85(\mathrm{~s}, 3 \mathrm{H}), 3.61(\mathrm{~s}, 3 \mathrm{H}), 2.58(\mathrm{~s}, 3 \mathrm{H}), 2.28(\mathrm{~s}$, $3 \mathrm{H}), 1.04(\mathrm{t}, J=7.1 \mathrm{~Hz}, 3 \mathrm{H}) \mathrm{ppm} ;{ }^{13} \mathrm{C}\left\{{ }^{1} \mathrm{H}\right\} \mathrm{NMR}\left(100 \mathrm{MHz}, \mathrm{CDCl}_{3}\right)$ : $\delta$ 168.7, 157.8, 155.4, 152.8, 151.6, 147.9, 142.3, 139.7, 133.6, 131.1, 128.8 (q, $\left.J_{\mathrm{C}-\mathrm{C}-\mathrm{F}}=32 \mathrm{~Hz}\right), 126.4,126.2,124.52,124.49,124.2$ $\left(\mathrm{q}, J_{\mathrm{C}-\mathrm{F}}=270 \mathrm{~Hz}\right), 122.4,108.4,61.3,61.1,61.0,56.1,24.2,23.1$, $13.8 \mathrm{ppm}$; IR (KBr): $\nu_{\max } 2935,1725,1586,1322,1260,1121,1084$, $1065,1005 \mathrm{~cm}^{-1}$; HRMS (ESI) $m / z$ : calcd for $\mathrm{C}_{26} \mathrm{H}_{27} \mathrm{~F}_{3} \mathrm{NO}_{5}[\mathrm{M}+\mathrm{H}]^{+}$ 490.1841, found: 490.1841 .

5-(4-Acetylphenyl)-2,6-dimethyl-3-(ethoxycarbonyl)-4-(3,4,5trimethoxyphenyl)pyridine (8m). White solid; $208 \mathrm{mg}, 45 \%$; $\operatorname{mp} 121{ }^{\circ} \mathrm{C} ;{ }^{1} \mathrm{H}$ NMR $\left(400 \mathrm{MHz}, \mathrm{CDCl}_{3}\right): \delta 7.81(\mathrm{~d}, J=8 \mathrm{~Hz}, 2 \mathrm{H})$, $7.34(\mathrm{~d}, J=7.3 \mathrm{~Hz}, 2 \mathrm{H}), 6.56(\mathrm{~s}, 1 \mathrm{H}), 6.41(\mathrm{~s}, 1 \mathrm{H}), 4.19-4.12(\mathrm{~m}$, $2 \mathrm{H}), 3.95(\mathrm{~s}, 3 \mathrm{H}), 3.85(\mathrm{~s}, 3 \mathrm{H}), 3.60(\mathrm{~s}, 3 \mathrm{H}), 2.58(\mathrm{~s}, 3 \mathrm{H}), 2.57(\mathrm{~s}$, $3 \mathrm{H}), 2.27(\mathrm{~s}, 3 \mathrm{H}), 1.05(\mathrm{t}, J=7.1 \mathrm{~Hz}, 3 \mathrm{H}) \mathrm{ppm} ;{ }^{13} \mathrm{C}\left\{{ }^{1} \mathrm{H}\right\} \mathrm{NMR}$ $\left(100 \mathrm{MHz}, \mathrm{CDCl}_{3}\right): \delta 198.0,168.7,157.8,155.3,152.8,151.6$, $148.0,142.3$, 141.1, 135.3, 133.5, 130.9, 127.7, 126.5, 126.3, 122.5, 108.4, 61.3, 61.11, 61.06, 56.1, 26.6, 24.3, 23.1, 13.8 ppm; IR (KBr): $\nu_{\max } 2924,2853,1725,1683,1586,1462,1262,1136$, 1085, $1005 \mathrm{~cm}^{-1}$; HRMS (ESI) $\mathrm{m} / z$ : calcd for $\mathrm{C}_{27} \mathrm{H}_{30} \mathrm{NO}_{6}[\mathrm{M}+$ $\mathrm{H}]^{+}$464.2073, found: 464.2082 .

5-(3-Acetylphenyl)-2,6-dimethyl-3-(ethoxycarbonyl)-4-(3,4,5trimethoxyphenyl)pyridine (8n). White solid; $199 \mathrm{mg}, 43 \%$; $\mathrm{mp}$ $129^{\circ} \mathrm{C} ;{ }^{1} \mathrm{H}$ NMR $\left(400 \mathrm{MHz}, \mathrm{CDCl}_{3}\right): \delta 7.84(\mathrm{~s}, 1 \mathrm{H}), 7.80-7.77(\mathrm{~m}$, $1 \mathrm{H}), 7.46(\mathrm{~d}, J=6.9 \mathrm{~Hz}, 1 \mathrm{H}), 7.32(\mathrm{dd}, J=7.7 \mathrm{~Hz}, J=7.6 \mathrm{~Hz} 1 \mathrm{H})$, $6.58(\mathrm{~s}, 1 \mathrm{H}), 6.43(\mathrm{~s}, 1 \mathrm{H}), 4.19-4.14(\mathrm{~m}, 2 \mathrm{H}), 3.96(\mathrm{~s}, 3 \mathrm{H}), 3.86(\mathrm{~s}$, $3 \mathrm{H}), 3.62(\mathrm{~s}, 3 \mathrm{H}), 2.56(\mathrm{~s}, 3 \mathrm{H}), 2.46(\mathrm{~s}, 3 \mathrm{H}), 2.27(\mathrm{~s}, 3 \mathrm{H}), 1.07(\mathrm{t}, J$ $=7.1 \mathrm{~Hz}, 3 \mathrm{H}) \mathrm{ppm} ;{ }^{13} \mathrm{C}\left\{{ }^{1} \mathrm{H}\right\} \mathrm{NMR}\left(100 \mathrm{MHz}, \mathrm{CDCl}_{3}\right): \delta$ 198.1, 168.7 , 157.8, 155.1, 152.6, 151.6, 148.0, 142.3, 136.4, 136.2, 135.4, 133.6, 131.2, 127.9, 126.6, 126.48, 126.45, 122.5, 108.4, $61.3,61.1,61.0,56.1,26.6,24.2,22.9,13.8 \mathrm{ppm}$; IR (KBr): $\nu_{\max }$ 2934, 2850, 1723, 1684, 1584, 1261, 1137, 1083, $1012 \mathrm{~cm}^{-1}$; HRMS (ESI) $m / z$ : calcd for $\mathrm{C}_{27} \mathrm{H}_{30} \mathrm{NO}_{6}[\mathrm{M}+\mathrm{H}]^{+}$464.2073, found: 464.2064 .

2,6-Dimethyl-3-(ethoxycarbonyl)-5-(3-hydroxyphenyl)-4-(3,4,5trimethoxyphenyl)pyridine (8o). Pale yellow solid; $175 \mathrm{mg}, 40 \%$; mp $169{ }^{\circ} \mathrm{C} ;{ }^{1} \mathrm{H}$ NMR $\left(400 \mathrm{MHz}, \mathrm{CDCl}_{3}\right.$ ): $\delta 7.05$ (s, 1H), 6.79-6.72 $(\mathrm{m}, 2 \mathrm{H}), 6.64(\mathrm{~d}, J=7.9 \mathrm{~Hz}, 1 \mathrm{H}), 6.53(\mathrm{~s}, 1 \mathrm{H}), 6.48(\mathrm{~s}, 1 \mathrm{H}), 4.14-$ $4.08(\mathrm{~m}, 2 \mathrm{H}), 3.94(\mathrm{~s}, 3 \mathrm{H}), 3.83(\mathrm{~s}, 3 \mathrm{H}), 3.61(\mathrm{~s}, 3 \mathrm{H}), 2.55(\mathrm{~s}, 3 \mathrm{H})$, $2.27(\mathrm{~s}, 3 \mathrm{H}), 1.01(\mathrm{t}, J=7.1 \mathrm{~Hz}, 3 \mathrm{H}) \mathrm{ppm} ;{ }^{13} \mathrm{C}\left\{{ }^{1} \mathrm{H}\right\} \mathrm{NMR}(100 \mathrm{MHz}$, $\left.\mathrm{CDCl}_{3}\right): \delta 168.8,157.4,155.5,154.9,152.3,151.7,148.8,142.3$, 137.1, 133.3, 128.8, 127.6, 126.5, 122.9, 117.8, 114.2, 108.2, 61.3, 
61.1, 61.0, 56.1, 23.8, 22.6, 13.7 ppm; IR (KBr): $\nu_{\max } 3395,2936$, 2851, 1726, 1592, 1448, 1280, 1133, $1096 \mathrm{~cm}^{-1}$; HRMS (ESI) $\mathrm{m} / \mathrm{z}$ : calcd for $\mathrm{C}_{25} \mathrm{H}_{28} \mathrm{NO}_{6}[\mathrm{M}+\mathrm{H}]^{+}$438.1916, found: 438.1916.

2,6-Dimethyl-3-(ethoxycarbonyl)-5-(4-(hydroxymethyl)phenyl)4-(3,4,5-trimethoxyphenyl)pyridine (8p). White solid; $203 \mathrm{mg}$, $45 \%$; mp $152{ }^{\circ} \mathrm{C} ;{ }^{1} \mathrm{H}$ NMR $\left(400 \mathrm{MHz}, \mathrm{CDCl}_{3}\right): \delta 7.23-7.17(\mathrm{~m}, 4 \mathrm{H})$, $6.54(\mathrm{~s}, 1 \mathrm{H}), 6.44(\mathrm{~s}, 1 \mathrm{H}), 4.64(\mathrm{~s}, 2 \mathrm{H}), 4.15-4.12(\mathrm{~m}, 2 \mathrm{H}), 3.94(\mathrm{~s}$, $3 \mathrm{H}), 3.84(\mathrm{~s}, 3 \mathrm{H}), 3.59(\mathrm{~s}, 3 \mathrm{H}), 2.56(\mathrm{~s}, 3 \mathrm{H}), 2.26(\mathrm{~s}, 3 \mathrm{H}), 1.03(\mathrm{t}, J=$ $7.1 \mathrm{~Hz}, 3 \mathrm{H}) \mathrm{ppm} ;{ }^{13} \mathrm{C}\left\{{ }^{1} \mathrm{H}\right\} \mathrm{NMR}\left(100 \mathrm{MHz}, \mathrm{CDCl}_{3}\right): \delta 168.7,157.5$, 155.2, 152.3, 151.7, 148.5, 142.2, 139.4, 134.9, 133.6, 130.8, 127.5, 126.4, 126.2, 122.7, 108.2, 64.9, 61.2, 61.1, 60.9, 56.1, 24.2, 22.9, $13.8 \mathrm{ppm}$; IR (KBr): $\nu_{\max } 3395,2980,2936,2850,1725,1588,1488$, $1267,1136,1094 \mathrm{~cm}^{-1}$; HRMS (ESI) $m / z$ : calcd for $\mathrm{C}_{26} \mathrm{H}_{30} \mathrm{NO}_{6}[\mathrm{M}$ $+\mathrm{H}]^{+}$452.2073, found: 452.2073.

2,6-Dimethyl-3-(ethoxycarbonyl)-5-(3-pyridinyl)-4-(3,4,5-trimethoxyphenyl)pyridine (8q). White solid; $177 \mathrm{mg}, 42 \%$; mp $107^{\circ} \mathrm{C}$; ${ }^{1} \mathrm{H}$ NMR (400 MHz, $\left.\mathrm{CDCl}_{3}\right): \delta 8.48(\mathrm{~s}, 1 \mathrm{H}), 8.42(\mathrm{dd}, J=4.8 \mathrm{~Hz}, J=$ $1.5 \mathrm{~Hz}, 1 \mathrm{H}), 7.53$ (d, $J=7.6 \mathrm{~Hz}, 1 \mathrm{H}), 7.15(\mathrm{dd}, J=7.6 \mathrm{~Hz}, J=$ $4.9 \mathrm{~Hz}, 1 \mathrm{H}), 6.58(\mathrm{~s}, 1 \mathrm{H}), 6.43(\mathrm{~s}, 1 \mathrm{H}), 4.18-4.09(\mathrm{~m}, 2 \mathrm{H}), 3.96(\mathrm{~s}$, $3 \mathrm{H}), 3.86(\mathrm{~s}, 3 \mathrm{H}), 3.64(\mathrm{~s}, 3 \mathrm{H}), 2.57(\mathrm{~s}, 3 \mathrm{H}), 2.30(\mathrm{~s}, 3 \mathrm{H}), 1.03(\mathrm{t}, J=$ $7.1 \mathrm{~Hz}, 3 \mathrm{H}) \mathrm{ppm} ;{ }^{13} \mathrm{C}\left\{{ }^{1} \mathrm{H}\right\} \mathrm{NMR}\left(100 \mathrm{MHz}, \mathrm{CDCl}_{3}\right): \delta 168.6,157.9$, 155.5, 152.9, 151.8, 151.3, 147.9, 147.7, 142.3, 138.0, 133.9, 131.7, 126.4, 123.8, 122.6, 122.5, 108.4, 61.3, 61.1, 61.0, 56.1, 24.3, 23.1, 13.8 ppm; IR (KBr): $\nu_{\max } 2978,2936,2848,1725,1586,1465,1385$, 1267, 1139, 1095, $1003 \mathrm{~cm}^{-1}$; HRMS (ESI) $\mathrm{m} / z$ : calcd for $\mathrm{C}_{24} \mathrm{H}_{27} \mathrm{~N}_{2} \mathrm{O}_{5}[\mathrm{M}+\mathrm{H}]^{+}$423.1920, found: 423.1918 .

2,6-Dimethyl-3-(ethoxycarbonyl)-5-(3-hydroxy-4-methoxyphenyl)-4-(3,4,5-trimethoxyphenyl)pyridine (8r). White solid; $210 \mathrm{mg}, 45 \%$; mp $148{ }^{\circ} \mathrm{C} ;{ }^{1} \mathrm{H}$ NMR (400 MHz, $\mathrm{CDCl}_{3}$ ): $\delta 6.85(\mathrm{~s}, 1 \mathrm{H}), 6.68-6.65(\mathrm{~m}, 2 \mathrm{H}), 6.51(\mathrm{~s}, 1 \mathrm{H}), 6.49(\mathrm{~s}, 1 \mathrm{H})$, $5.61(\mathrm{~s}, 1 \mathrm{H}), 4.14-4.09(\mathrm{~m}, 2 \mathrm{H}), 3.93(\mathrm{~s}, 3 \mathrm{H}), 3.84(\mathrm{~s}, 3 \mathrm{H}), 3.83$ $(\mathrm{s}, 3 \mathrm{H}), 3.62(\mathrm{~s}, 3 \mathrm{H}), 2.57(\mathrm{~s}, 3 \mathrm{H}), 2.32(\mathrm{~s}, 3 \mathrm{H}), 1.01(\mathrm{t}, J=$ $7.1 \mathrm{~Hz}, 3 \mathrm{H}) \mathrm{ppm} ;{ }^{13} \mathrm{C}\left\{{ }^{1} \mathrm{H}\right\} \mathrm{NMR}\left(100 \mathrm{MHz}, \mathrm{CDCl}_{3}\right): \delta 168.8$, 157.5, 155.2, 152.1, 151.9, 148.8, 145.6, 144.8, 142.2, 133.7, $128.9,127.4,126.3,122.7,122.6,117.0,109.9,108.1,61.1$, $61.0,60.9,56.1,55.8,24.2,23.0,13.7 \mathrm{ppm}$; IR (KBr): $\nu_{\max }$ 3410, 2935, 2838, 1721, 1586, 1488, 1250, 1087, $1015 \mathrm{~cm}^{-1}$; HRMS (ESI) $m / z$ : calcd for $\mathrm{C}_{26} \mathrm{H}_{30} \mathrm{NO}_{7}[\mathrm{M}+\mathrm{H}]^{+}$468.2022, found: 468.2013 .

4-(3-(Benzyloxy)-4-methoxyphenyl)-2,6-dimethyl-3-(ethoxycarbonyl)-5-(3,4,5-trimethoxyphenyl)nicotinate (8s). Yellow solid; $250 \mathrm{mg}, 45 \%$; $\mathrm{mp} 120{ }^{\circ} \mathrm{C} ;{ }^{1} \mathrm{H}$ NMR $\left(400 \mathrm{MHz}, \mathrm{CDCl}_{3}\right): \delta 7.46(\mathrm{~d}, J$ $=8.6 \mathrm{~Hz}, 2 \mathrm{H}), 7.38(\mathrm{dd}, J=8.6 \mathrm{~Hz}, J=7.1 \mathrm{~Hz}, 2 \mathrm{H}), 7.32(\mathrm{dd}, J=$ $7.2 \mathrm{~Hz}, J=7.0 \mathrm{~Hz} \mathrm{1H}), 6.98(\mathrm{~s}, 1 \mathrm{H}), 6.83(\mathrm{~s}, 1 \mathrm{H}), 6.49(\mathrm{~s}, 3 \mathrm{H})$, 4.04-4.02 (m, 2H), 3.96 (s, 3H), $3.81(\mathrm{~s}, 3 \mathrm{H}), 3.67$ (s, 6H), 2.57 (s, $3 \mathrm{H}), 2.34(\mathrm{~s}, 3 \mathrm{H}), 1.01(\mathrm{t}, J=7.2 \mathrm{~Hz}, 3 \mathrm{H}) \mathrm{ppm} ;{ }^{13} \mathrm{C}\left\{{ }^{1} \mathrm{H}\right\} \mathrm{NMR}(100$ $\left.\mathrm{MHz}, \mathrm{CDCl}_{3}\right): \delta 168.8,157.9,154.7,152.7,149.4,148.8,147.1$, 136.9, 136.7, 135.8, 133.6, 129.3, 128.6, 127.9, 127.4, 126.7, 122.7, $114.8,113.4,106.9,71.1,61.3,60.9,56.2,56.0,24.3,22.9$, 13.9 ppm; IR (KBr): $\nu_{\max } 2936,2840,1715,1586,1501,1255$, $1122,1079 \mathrm{~cm}^{-1}$; HRMS (ESI) $m / z$ : calcd for $\mathrm{C}_{33} \mathrm{H}_{36} \mathrm{NO}_{7}[\mathrm{M}+\mathrm{H}]^{+}$ 558.2492, found: 558.2476 .

2,6-Dimethyl-3-(ethoxycarbonyl)-4-(3-hydroxy-4-methoxyphenyl)-5-(3,4,5-trimethoxyphenyl)pyridine (8t). White solid; $350 \mathrm{mg}$, 75\%; $\mathrm{mp}>200{ }^{\circ} \mathrm{C} ;{ }^{1} \mathrm{H} \mathrm{NMR}\left(400 \mathrm{MHz}, \mathrm{CDCl}_{3}\right)$ : $\delta 6.94(\mathrm{~s}, 1 \mathrm{H}), 6.82(\mathrm{~s}, 1 \mathrm{H}), 6.51-6.48(\mathrm{~m}, 3 \mathrm{H}), 5.78(\mathrm{br} \mathrm{s}, 1 \mathrm{H})$, 4.18-4.13 (m, 2H), $3.96(\mathrm{~s}, 3 \mathrm{H}), 3.80(\mathrm{~s}, 3 \mathrm{H}), 3.67(\mathrm{~s}, 6 \mathrm{H}), 2.56$ (s, 3H), $2.34(\mathrm{~s}, 3 \mathrm{H}), 1.12(\mathrm{t}, J=7.1 \mathrm{~Hz}, 3 \mathrm{H}) \mathrm{ppm} ;{ }^{13} \mathrm{C}\left\{{ }^{1} \mathrm{H}\right\}$ NMR (100 MHz, $\mathrm{CDCl}_{3}$ ): $\delta 168.7,157.8,154.6,152.6,148.8$, $146.5,144.7,136.8,136.0,132.6,129.9$, 126.8, 122.7, 115.6, 112.2, 106.9, 61.3, 60.9, 56.1, 56.0, 24.2, 22.8, 13.9 ppm; IR (KBr): $\nu_{\max } 3453,2961,2935,2840,1725,1587,1499,1260$, $1125,1083,1018 \mathrm{~cm}^{-1}$; HRMS (ESI) $\mathrm{m} / z$ : calcd for $\mathrm{C}_{26} \mathrm{H}_{30} \mathrm{NO}_{7}$ $[\mathrm{M}+\mathrm{H}]^{+}$468.2022, found: 468.2015 .

\section{Biology}

Cell-based screening assay. Synthesized penta-substituted/ functionalized-3,4-diarylated pyridine compounds 8a-8t used in this study were dissolved in $100 \%$ DMSO (cell culture grade, Himedia). The compounds were serially diluted in Dulbecco's Modified Eagle (DMEM) cell culture medium to maintain the DMSO concentration less than $0.1 \%$ for analyzing its cell proliferation inhibiting potency in HeLa cell line. Briefly, $5 \times$ $10^{3}$ cells were seeded in 96 well plate. ${ }^{15 b}$ After $16 \mathrm{~h}$ compounds $(5 \mu \mathrm{M})$ were added and incubated with cells for $24 \mathrm{~h}$. Subsequently, cells were fixed with TCA and processed for sulforhodamine B (SRB) assay. ${ }^{39}$

Effects of diarylated pyridine compounds on in vitro tubulin assembly. Tubulin was purified from goat brain using $1 \mathrm{M}$ glutamate as described earlier. ${ }^{\mathbf{4 0 , 4 1}}$ Tubulin concentration was determined by Bradford method. ${ }^{42}$ Purified tubulin $(12 \mu \mathrm{M})$ in PEM buffer (25 mM PIPES pH 6.8, $3 \mathrm{mM} \mathrm{MgCl}_{2}$ and $1 \mathrm{mM}$ EGTA) was incubated in the absence and presence of $20 \mu \mathrm{M}$ of compounds (8b, 8f, 8j and 8p) for $10 \mathrm{~min}$ on ice and then, DMSO (final concentration 10\%) and $1 \mathrm{mM}$ GTP was added to the reaction mixtures. Subsequently, the assembly kinetics was monitored at $37{ }^{\circ} \mathrm{C}$ by $90^{\circ}$ light scattering $(350 \mathrm{~nm})$ using Spectramax $\mathrm{M} 2{ }^{\mathrm{e}}$. The extent of inhibition of polymerization was measured after $30 \mathrm{~min}$ of assembly. The light scattering data of only compounds $(20 \mu \mathrm{M})$ were also recorded and subtracted from their respective data set. Three independent set of experiments were performed.

\section{Acknowledgements}

We gratefully acknowledge financial support from CSIR, New Delhi for this investigation. NH is thankful to DST, NewDelhi for her DST-INSPIRE fellowship. DP is thankful to DAE, Government of India for DAE-SRC fellowship.

\section{Notes and references}

1 (a) A. O. Plunkett, Nat. Prod. Rep., 1994, 11, 581; (b) J. Daly, H. Garaffo and T. Spande, Alkaloids, 1993, 43, 185; (c) A. R. Pinder, Nat. Prod. Rep., 1992, 9, 491; (d) G. D. Henry, Tetrahedron, 2004, 60, 6043; (e) J. P. Michael, Nat. Prod. Rep., 2005, 22, 627.

2 D. Riendeau, M. D. Percival, C. Brideau, S. Charleson, D. Dube, D. Ethier, J.-P. Falgueyret, R. W. Friesen, R. Gordon, G. Greig, J. Guay, J. Mancini, M. Ouellet, E. Wong, L. Xu, S. Boyce, D. Visco, Y. Girard, P. Prasit, R. Zamboni, I. W. Rodger, M. Gresser, A. W. Ford- 
Hutchinson, R. N. Young and C.-C. Chan, J. Pharmacol. Exp. Ther., 2001, 296, 558.

3 I. W. Davies, J.-F. Marcoux, E. G. Corley, M. Journet, D.-W. Cai, M. Palucki, J. Wu, R. D. Larsen, K. Rossen, P. J. Pye, L. DiMichele, P. Dormer and P. J. Reider, J. Org. Chem., 2000, 65, 8415.

4 R. Karki, P. Thapa, H. Y. Yoo, T. M. Kadayat, P.-H. Park, Y. Na, E. Lee, K.-H. Jeon, W.-J. Cho and H. Choi, Eur. J. Med. Chem., 2012, 49, 219.

5 T. Hanada, Y. Hashizume, N. Tokuhara, O. Takenaka, N. Kohmura, A. Ogasawara, S. Hatakeyama, M. Ohgoh, M. Ueno and Y. Nishizawa, Epilepsia, 2011, 52, 1331.

6 P. Eastwood, J. Gonzalez, S. Paredes, A. Nueda, T. Domenech, J. Alberti and B. Vidal, Bioorg. Med. Chem. Lett., 2010, 20, 1697.

7 R. H. Brad, H. D. David, A. J. Stacey, L. S. Eugene, L. W. Susan and W. W. Robert, Bioorg. Med. Chem. Lett., 2001, 11, 1939.

8 C. Pascard, A. Ducruix, J. Lunel and T. Prange, J. Am. Chem. Soc., 1977, 99, 6418.

9 M. C. Bagley, K. E. Bashford, C. L. Hesketh and C. J. Moody, J. Am. Chem. Soc., 2000, 122, 3301.

10 A. G. Fang, J. V. Mello and N. S. Finney, Tetrahedron, 2004, 60, 11075.

11 (a) S. Hang, D. J. Babinski and T. Ritter, J. Am. Chem. Soc., 2015, 137, 3775; (b) S. K. Guchhait and G. Priyadarshani, $J$. Org. Chem., 2015, 80, 8482; (c) S. K. Guchhait and G. Priyadarshani, J. Org. Chem., 2015, 80, 6342; (d) G. Priyadarshani, S. Amrutkar, A. Nayak, U. C. Banerjee, C. N. Kundu and S. K. Guchhait, Eur. J. Med. Chem., 2016, 122, 43.

12 H. Sekizawa, K. Amaike, Y. Itoh, T. Suzuki, K. Itami and J. Yamaguchi, ACS Med. Chem. Lett., 2014, 5, 582.

13 For representative activities, e.g., antimitotic, anitproliferative, antimalarial, fungicidal: (a) C.-J. Chen, B.-A. Song, S. Yang, G.-F. Xu, P. S. Bhadury, L.-H. Jin, D.-Y. Hu, Q.-Z. Li, F. Liu and W. Xue, Bioorg. Med. Chem., 2007, 15, 3981; (b) R. Kumar, D. Mohanakrishnan, A. Sharma, N. K. Kaushik, K. Kalia, A. K. Sinha and D. Sahal, Eur. J. Med. Chem., 2010, 45, 5292; (c) Y.-H. Wang, M. Goto, L.-T. Wang, K.-Y. Hsieh, S. L. MorrisNatschke, G.-H. Tang, C.-L. Long and K.-H. Lee, Bioorg. Med. Chem. Lett., 2014, 24, 4818; (d) M. Cushman, D. Nagarathnam, D. Gopal, H. M. He, C. M. Lin and E. Hamel, J. Med. Chem., 1992, 35, 2293.

14 (a) G. R. Pettit, S. B. Singh, M. R. Boyd, E. Hamel, R. K. Pettit, J. M. Schmidt and F. Hogan, J. Med. Chem., 1995, 38, 1666; (b) C. Canela, R. M. Moraesb, F. E. Dayana and D. Ferreirab, Phytochemistry, 2000, 54, 115.

15 (a) A. T. Baviskar, C. Madaan, R. Preet, P. Mohapatra, V. Jain, A. Agarwal, S. K. Guchhait, C. N. Kundu, U. C. Banerjee and P. V. Bharatam, J. Med. Chem., 2011, 54, 5013; (b) V. Chaudhary, J. B. Venghateri, H. P. Dhaked, A. S. Bhoyar, S. K. Guchhait and D. Panda, J. Med. Chem., 2016, 59, 3439; (c) G. Priyadarshani, A. Nayak, S. M. Amrutkar, S. Das, S. K. Guchhait, C. N. Kundu and U. C. Banerjee, ACS Med. Chem. Lett., 2016, 7, 1056.
16 V. Leuranguer, M. E. Mangoni, J. Nargeot and S. Richard, J. Cardiovasc. Pharmacol. Ther., 2001, 37, 649.

17 P. D. Boatman, J. G. Richman and G. Semple, J. Med. Chem., 2008, 51, 7653.

18 (a) R. Abramovitch and E. Smith, Chemistry of heterocyclic compounds: pyridine and its derivatives: part II, John Wiley \& Sons, Inc, New York, 1974; (b) G. Newkome, Part 5, Chemistry of Heterocyclic Compounds: Pyridine and its Derivatives, Part 5, Wiley \& Sons, Hoboken, NJ, USA, 1984.

19 (a) E. Zhang, J. Tang, S. Li, P. Wu, J. E. Moses and K. B. Sharpless, Chem.-Eur. J., 2016, 22, 5692; (b) Z. Song, X. Huang, W. Yi and W. Zhang, Org. Lett., 2016, 18, 5640; (c) J. Shen, D. Cai, C. Kuai, Y. Liu, M. Wei, G. Cheng and X. Cui, J. Org. Chem., 2015, 80, 6584; (d) R. Khajuria, P. Kannaboina, K. K. Kapoor, A. Gupta, G. Raina, A. K. Jassal, L. K. Rana, M. S. Hundald and P. Das, Org. Biomol. Chem., 2015, 13, 5944.

20 (a) R. L. Frank and R. P. Seven, J. Am. Chem. Soc., 1949, 71, 2629; (b) B. Heller and M. Hapke, Chem. Soc. Rev., 2007, 36, 1085; (c) H. Bönnemann, Angew. Chem., Int. Ed. Engl., 1978, 17, 505; (d) M. Ohashi, I. Takeda, M. Ikawa and S. Ogoshi, J. Am. Chem. Soc., 2011, 133, 18018; (e) I. Linder, M. Gerhard, L. Schefzig, M. Andrä, C. Bentz, H. U. Reissig and R. Zimmer, Eur. J. Org. Chem., 2011, 2011, 6070; (f) C. Allais, F. Liéby-Muller, T. Constantieux and J. Rodriguez, Adv. Synth. Catal., 2012, 354, 2537; $(g)$ S.-I. Yamamoto, K. Okamoto, M. Murakoso, Y. Kuninobu and K. Takai, Org. Lett., 2012, 14, 3182.

21 (a) K. Billingsley and S. L. Buchwald, J. Am. Chem. Soc., 2007, 129, 3358-3366; (b) K. L. Billingsley, K. W. Anderson and S. L. Buchwald, Angew. Chem., Int. Ed., 2006, 45, 3484; (c) G. M. Chapman, S. P. Stanforth, B. Tarbit and M. D. Watson, J. Chem. Soc., Perkin Trans. 1, 2002, 581; (d) S. Reimann, S. Parpart, P. Ehlers, M. Sharif, A. Spannenbergb and P. Langer, Org. Biomol. Chem., 2015, 13, 6832; (e) W. Hagui, N. Besbes, E. Srasra, J.-F. Soulé and H. Doucet, $R S C A d v$., 2016, 6, 17110; (f) S. Reimann, P. Ehlers, S. Parpart and A. Surkus, Tetrahedron, 2015, 71, 5371.

22 (a) C. Marti, J. Irurre, A. Alvarez-Larena, J. F. Piniella, E. Brillas, L. Fajari, C. Aleman and L. Julia, J. Org. Chem., 1994, 59, 6200; (b) A. Schmidt, T. Mordhorst and M. Nieger, Org. Biomol. Chem., 2005, 3, 3788; (c) G. Sandford, R. Slater, D. S. Yufit, J. A. K. Howard and A. Vong, J. Org. Chem., 2005, 70, 7208.

23 (a) F. Mongin and G. Quéguiner, Tetrahedron, 2001, 57, 4059; (b) P. C. Gros and Y. Fort, Eur. J. Org. Chem., 2009, 2009, 4199. 24 A. Debache, R. Boulcina, A. Belfaitah, S. Rhouati and B. Carnobi, Synlett, 2008, 4, 509.

25 (a) R. S. Varma and D. Kumar, Tetrahedron Lett., 1999, 40, 21; (b) X. Liao, W. Lin, J. Lu and C. Wang, Tetrahedron Lett., 2010, 51, 3859; (c) M. M. Heravi, F. K. Behbahani, H. A. Oskooie and R. H. Shoar, Tetrahedron Lett., 2005, 46, 2775 .

26 (a) J.-J. Dai, J.-H. Liu, D.-F. Luo and L. Liu, Chem. Commun., 2011, 47, 677; (b) F. A. Arroyave and J. R. Reynolds, J. Org. Chem., 2011, 76, 8621; (c) R. Shang, Z.-W. Yang, Y. Wang, 
S.-L. Zhang and L. Liu, J. Am. Chem. Soc., 2010, 132, 14391; (d) P. Forgione, M.-C. Brochu, M. St-Onge, K. H. Thesen, M. D. Bailey and F. Bilodeau, J. Am. Chem. Soc., 2006, 128, 11350.

27 (a) B. Love and M. M. Goodman, J. Heterocycl. Chem., 1975, 12, 363; (b) L. J. Gooßen, C. Linder, N. Rodríguez, P. P. Lange and A. Fromm, Chem. Commun., 2009, 7173; (c) R. Meesala, M. N. Mordi and S. M. Mansor, Synlett, 2014, 25, 120; (d) J. Cornella, C. Sanchez, D. Banawa and I. Larrosa, Chem. Commun., 2009, 7176; (e) P. Lu, C. Sanchez, J. Cornella and I. Larrosa, Org. Lett., 2009, 11, 5710 .

28 M. Ye, G.-L. Gao, A. J. F. Edmunds, P. A. Worthington, J. A. Morris and J.-Q. Yu, J. Am. Chem. Soc., 2011, 133, 19090. 29 F. Dai, Q. Gui, J. Liu, Z. Yang, X. Chen, R. Guo and Z. Tan, Chem. Commun., 2013, 49, 4634.

30 S. K. Guchhait, S. Kandekar, M. Kashyap, N. Taxak and P. V. Bharatam, J. Org. Chem., 2012, 77, 8321.

31 M. V. Adhikari and S. D. Samant, Ultrason. Sonochem., 2002, 9, 107.

32 C. Liu, N. Han, X. Song and J. Qiu, Eur. J. Org. Chem., 2010, 2010, 5548.

33 M. Christakakou, M. Schön, M. Schnürch and M. D. Mihovilovic, Synlett, 2013, 24, 2411.

34 (a) Y. Hayashi, Chem. Sci., 2016, 7, 866; (b) C. Vaxelaire, P. Winter and M. Christmann, Angew. Chem., Int. Ed.,
2011, 50, 3605; (c) C. Liu, Z. Zeng, R. Chen, X. Jiang, Y. Wang and Y. Zhang, Org. Lett., 2016, 18, 624; (d)

A. Shavnya, S. B. Coffey, K. D. Hesp, S. C. Ross and A. S. Tsai, Org. Lett., 2016, 18, 5848.

35 D. Simoni, G. Grisolia, G. Giannini, M. Roberti, R. Rondanin,

L. Piccagli, R. Baruchello, M. Rossi, R. Romagnoli, F. P. Invidiata, S. Grimaudo, M. K. Jung, E. Hamel, N. Gebbia, L. Crosta, V. Abbadessa, A. D. Cristina, L. Dusonchet, M. Meli and M. Tolomeo, J. Med. Chem., 2005, 48, 723.

36 S. Zheng, Q. Zhong, M. Mottamal, Q. Zhang, C. Zhang, E. LeMelle, H. McFerrin and G. Wang, J. Med. Chem., 2014, 57, 3369.

37 T. Ishiyama, M. Murata and N. Miyaura, J. Org. Chem., 1995, 60, 7508.

38 T. D. A. Kumar, P. Mohan, C. V. S. Subrahmanyam and K. Satyanarayana, Synth. Commun., 2014, 44, 574.

39 P. Skehan, R. Storeng, D. Scudiero, A. Monks, J. McMahon,

D. Vistica, J. T. Warren, H. Bokesch, S. Kenney and M. R. Boyd, J. Natl. Cancer Inst., 1990, 82, 1107.

40 E. Hamel and C. M. Lin, Arch. Biochem. Biophys., 1981, 209, 29.

41 D. Panda, K. Rathinasamy, M. K. Santra and L. Wilson, Proc. Natl. Acad. Sci. U. S. A., 2005, 102, 9878.

42 M. M. Bradford, Anal. Biochem., 1976, 72, 248. 